\title{
C/EBPS demonstrates a dichotomous role in tumor initiation and promotion of epithelial carcinoma
}

Ramlogan Sowamber ${ }^{1,2}$, Rania Chehade ${ }^{1,2}$, Mahmoud Bitar ${ }^{1,2}$, Leah Dodds ${ }^{5,7}$, Anca Milea ${ }^{1,2}$, Brian Slomovitz $^{, 5,6,7}$, Patricia A Shaw ${ }^{1,2,3,4}$ and Sophia HL George ' $^{1,2,5,6,7}$

Campbell Family Institute for Breast Cancer Research ${ }^{1}$,

Princess Margaret Cancer Center, University Health Network ${ }^{2}$

Department of Laboratory Medicine and Pathobiology ${ }^{3}$, University of Toronto ${ }^{4}$

Sylvester Comprehensive Cancer Center ${ }^{5}$

Department of Obstetrics and Gynecology, Division of Gynecology Oncology ${ }^{6}$,

University of Miami, Leonard Miller School of Medicine ${ }^{7}$

Corresponding author:

Sophia HL George*

sophia.george@med.miami.edu

Now at Division of Gynecologic Oncology, Sylvester Comprehensive Cancer Center, Leonard Miller School of Medicine, University of Miami 1550 NW $10^{\text {th }}$ Ave, Room 414 (M-877), Miami, FL, 33136

Running Title: C/EBP $\delta$ 's dichotomous role in high-grade serous ovarian cancer The authors disclose no potential conflicts of interest.

Key Words 


\section{Abstract}

$39 C / E B P \delta$ (CEBPD), a gene part of the highly conserved basic-leucine zipper (b-ZIP) domain of

40 transcriptional factors, is downregulated in $65 \%$ of high grade serous carcinoma of the ovary (HGSC).

41 Overexpression of $C / E B P \delta$ in different tumors as glioblastoma and breast cancer either promotes tumor

42 progression or inhibits growth. Despite these contradictory roles in different cancer types, we show that

$43 C / E B P \delta$ overexpression has a consistent function of downregulating proliferation and promoting

44 migration in fallopian tube epithelial cells (FTE). We show that the FTE have both mesenchymal and

45 epithelial cell characteristics. Further, our data supports a role for $C / E B P \delta$ as an early regulatory

46 transcriptional factor that promotes a mesenchymal to epithelial (MET) phenotype by upregulating E-

47 cadherin and downregulating vimentin and N-cadherin in FTE cells. We demonstrate that

48 overexpression of $C / E B P \delta$ in ovarian and breast cancer cell lines have consistent effects and phenotype

49 as the FTE cells. Our findings suggest a role for $C / E B P \delta$ in the early events of ovarian serous

50 carcinogenesis which may be used to help further understand how the disease develops from a

51 premalignant cells.

52

53

54

55

56

57

58

59

60 


\section{Introduction}

62 High-grade serous ovarian cancer (HGSC) remains the most fatal gynecological malignancy and

63 accounts for the majority of deaths due to ovarian cancer [1]. Improving early detection, prevention and

64 overall prognosis was, limited by a lack of understanding of the etiology of HGSC. Now, significant

65 data suggests the distal end of the fallopian tube is the site of origin for HGSC [2]. Detailed

66 histopathological examination of the fallopian tube epithelium in $B R C A$ mutation carriers undergoing

67 prophylactic bilateral salpingo-oophorectomy led to the identification of precursor lesions [ㄹ, $\underline{4}]$.

69 A pre-neoplastic lesion called the p53 signature is the earliest mutational and genomic event described in the gradual steps of HGSC development [5]. The acquisition of somatic TP53 mutations is followed

71 by additional genomic alterations, cellular tufting and loss of polarity, resulting in the development of neoplastic serous tubal intraepithelial carcinoma (STIC) [6-12]. STIC lesions share multiple genomic copy number alterations, along with mutations in tumor suppressors and oncogenes, including TP53,

BRCA1 and BRCA2, RB1, STK11, FOXO3a, CCNE1, STATHMIN1 and hTERT, which are observed

prior to HGSC metastasis to the ovaries and peritoneal spread [으, 10, $\underline{13-19}]$. In this model, cells that exfoliate from the fallopian tube into the peritoneal cavity must evade anoikis and detachment associated apoptosis before attaching to the mesothelial ovarian surface $[\underline{20}, \underline{21}]$.

79 The fallopian tube epithelia (FTE) undergo monthly cycles of hormonally driven proliferation and 80 differentiation. In a previous study, we identified CCAAT/enhancer binding protein delta $(C / E B P \delta)$, to 81 be transcriptionally upregulated in the FTE of BRCAl mutation carriers and in the post-ovulatory 82 (luteal) phase of the ovarian cycle a process linked to cytotoxic stress [22]. C/EBP $\delta$ is located on 83 chromosome 8q11.21 and belongs to the superfamily of highly conserved basic-leucine zipper (b-ZIP) 
84 domain transcriptional factors [23]. It has multiple functions related to inflammation, cell cycle

85 regulation, differentiation and metabolism [22-25]. Although C/EBP $\delta$ overexpression promotes

86 glioblastoma progression and is associated with poor progression in pancreatic and urothelial cancers

87 [26,$\underline{27}]$, its overexpression in breast, prostate and myeloid cancers, inhibits growth and promotes

88 differentiation [28-30]. Furthermore, low expression of C/EBP $\delta$ was reported in cervical, hepatocellular

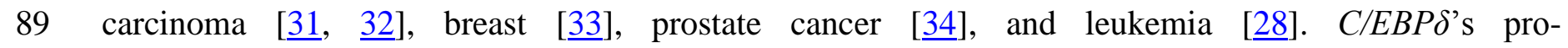

90 oncogenic/tumor suppressive function is cell type and context dependent [26, $\underline{31}, \underline{35-37]}$. Little is known

91 about the role of $C / E B P \delta$ in the development of HGSC. The objectives of this study were to explore

92 expression of $C / E B P \delta$ in HGSC tumors as well as precursor lesions and determine the effects of

$93 C / E B P \delta$ on FTE cancer cell growth and migration.

\section{Results}

\section{$96 \mathbf{C} / \mathbf{E B P} \delta$ is differentially expressed across serous ovarian cancer histotypes}

97 We previously reported higher expression levels of $\mathrm{C} / \mathrm{EBP} \delta$ at the mRNA and protein levels in the luteal 98 phase of the normal fallopian tube epithelia [22]. To investigate C/EBP $\delta$ protein expression levels across 99 serous ovarian cancer histotypes, immunohistochemistry was performed on a cohort of 366 high grade 100 serous carcinoma (HGSC) and 26 low-grade serous carcinoma (LGSC) on 5 independent tissue 101 microarrays (TMAs). Each core was annotated to include epithelia and exclude stroma. Mean intensity 102 and percent positivity using a nuclear stain algorithm was reported based on the following histoscores: 103 no expression (0); low (+1); medium (+2) and high (+3) (Figure 1A). In FTE, C/EBP $\delta$ is expressed in 104 the nuclei of secretory and ciliated cells. Seventy-six per cent $(76.5 \%, 280 / 366)$ of HGSC cases had low 105 or attenuated (histoscore of $0 /+1) \mathrm{C} / \mathrm{EBP} \delta$ protein expression whereas $24 \%(86 / 366)$ had a medium to 106 high expression (histoscore of $+2 /+3)$. Seventy-six per cent $(76.9 \%, 20 / 26)$ of LGSC cases had medium 
107 to high expression compared to $23 \%$ (6/26) with low C/EBP $\delta$ expression. Overall, HGSC had 2-fold 108 lower $\mathrm{C} / \mathrm{EBP} \delta$ protein expression relative to LGSC ( $\mathrm{p}=0.0004)$ (Figure 1B).

110 To determine if $\mathrm{C} / \mathrm{EBP} \delta$ protein expression seen in HGSC is correlated to genomic changes, we used a 111 cohort of HGSC genomic data (Affymetrix 6.0 SNP array) of chemotherapy naïve and stage III/IV cases

$112(n=75) \quad[\underline{6}, \underline{13}]$. Seventeen per cent $(13 / 75)$ of cases $(p<0.0001)$ had an amplification of the gene 113 whereas only $2 \%(2 / 75)$ cases revealed a deletion; most cases (59/75) remained diploid at that genomic

114 locus (Supplementary Figure 1A). In the TCGA ovarian cancer dataset, [16], 2.5\% (8/316) of HGSC 115 cases had an amplification and 3.1\% (10/316) had a shallow deletion of $C / E B P \delta$ with few cases (less 116 than 10\%) having the mRNA downregulated (http://bit.ly/2DW9QPa) (Supplementary Figure 1A). 117 Patients with high $(+2 /+3) \mathrm{C} / \mathrm{EBP} \delta$ protein expression had no significant progression free survival (PFS, 118 Log rank test, $\mathrm{p}=0.319)$ or overall survival $(\mathrm{OS})(\log$ rank test, $\mathrm{p}=0.330)$ compared to low $(0 /+1)$ $119 \mathrm{C} / \mathrm{EBP} \delta$ protein expression levels (Supplementary Figure 1B).

121 Reduced C/EBP $\delta$ expression levels in STIC lesions reflect early changes observed in HGSC

122 Since $\mathrm{C} / \mathrm{EBP} \delta$ levels are higher in normal FTE and differentially expressed in HGSC, we assessed $123 \mathrm{C} / \mathrm{EBP} \delta$ expression in a small cohort of matched normal FTE, STIC and HGSC (n=13). Our data 124 revealed that $53.8 \%(7 / 13)$ of normal fallopian tube cases had higher C/EBP $\delta$ expression levels $(+2 /+3$ 125 expression) compared to other STIC's and HGSC, whereas in 46\% (6/13) of cases, C/EBP $\delta$ expression 126 was maintained between FTE and STIC. In normal FTE, high C/EBP $\delta$ expression was associated with 127 low proliferation as determined by Ki67 expression ( $\mathrm{p}=0.02)$. In STIC, however, C/EBP $\delta$ expression 128 was slightly lower than in normal FTE, while Ki67 expression increased relative to normal FTE 
$129(\mathrm{p}=0.08)$ and HGSCs. There were fewer $\mathrm{C} / \mathrm{EBP} \delta$ expressing cells in HGSC relative to highly

130 proliferative $\left(\mathrm{Ki}_{67}{ }^{+}\right)$and p53 expressing cells $(\mathrm{p}<0.0001)$ (Figure 1C-E). Overall, C/EBP $\delta$ expression

131 was inversely related to proliferation during the transition from normal FTE, to STIC and subsequently

132 HGSC. Given the results and the known role of $\mathrm{C} / \mathrm{EBP} \delta$ in cell cycle control, we sought to determine

133 whether overexpression of C/EBP $\delta$ would regulate FTE and cancer cell growth.

Overexpression of $\mathrm{C} / \mathrm{EBP} \delta$ inhibits proliferation in premalignant fallopian tube epithelia and

cancer cells.

137 Fallopian Tube Epithelia

138 To model p53 signatures in vitro, FTE cell lines with a p53-R175H dominant negative mutation $139(\mathrm{p} 53 \mathrm{DN})$ and human telomerase (hTERT) over-expression were generated [6] (Figure 2A). As expected 140 from immunohistochemical data, proliferating p53DN FTE cell lines had low levels of endogenous $141 \mathrm{C} / \mathrm{EBP} \delta$ protein (Figure 2B-C). A C/EBP $\delta$ gain-of-function model was generated with a lentivirus based $p C D H-C M V-G F P-P U R O$ expression vector, to examine its effects on proliferation of FTE cells. Three

143 independent FTE cell lines were transfected with either empty vector (FTE-ctrl) or $\mathrm{C} / \mathrm{EBP} \delta \mathrm{cDNA}$ 144 vector (FTE-CEBPD-OE) to generate stable lines (Figure 2A-B, Supplementary Figure 2B). C/EBP $\delta$ 145 overexpression was confirmed by immunofluorescence (localized to the nucleus) and by western blot 146 analyses (Figure 2B-C). To examine the role of $\mathrm{C} / \mathrm{EBP} \delta$ in cell cycle regulation, cell cycle analysis and 147 growth kinetics were performed on FTE-ctrl and FTE-CEBPD-OE cell lines. Over-expression of $148 \mathrm{C} / \mathrm{EBP} \delta$ reduced cell growth compared to controls, as measured by population doubling (PD) over time 149 (FTE19-CEBPD-OE versus control, 7.1-fold, $\mathrm{p}=0.04$; FTE57-CEBPD-OE vs control, 14.9-fold, $\mathrm{p}=0.01$; 150 each in triplicate) (Figure 2D, Supplementary Figure 2C). Cell cycle analysis by BrdU /propidium iodide 151 incorporation and flow cytometry in FTE cells demonstrated a propensity for C/EBP $\delta$ overexpressing 
152 cells to accumulate in the G1 phase of the cell cycle compared to FTE-ctrl cells ( $\mathrm{p}=0.0019)$ (Figure 2E).

$153 \mathrm{C} / \mathrm{EBP} \delta$ is known to interact with CCND1 and promotes STAT3 induced G0 cell cycle arrest [38,$\underline{39]}$. In

154 addition, overexpression of $\mathrm{C} / \mathrm{EBP} \delta$ decreased anchorage independent growth of FTE cells compared to 155 control cells (FTE19-p53DN-hTERT, 1.97-fold, p=0.0009; FTE57-p53DN-hTERT, 1.41-fold, p=0.07)

156 (Figure 2F), but increased colony formation in one cell line (Supplementary Figure 2D). Overall, the 157 results are consistent with decreased growth rates in $2 \mathrm{D}$ and $3 \mathrm{D}$ assays. The data show that $\mathrm{C} / \mathrm{EBP} \delta$ 158 overexpression is sufficient to inhibit proliferation in FTE cells likely at the G1/S phase of the cell cycle.

\section{Breast and ovarian cancer cell lines}

161 To determine C/EBP $\delta$ basal expression levels across ovarian $(n=42)$ and breast cancer $(n=54)$ cell lines,

162 we used publically available RNA-sequence data from Medrano et al. [40]. The data uniformly showed 163 higher $\mathrm{C} / \mathrm{EBP} \delta$ mRNA levels were associated with lower proliferation rates across breast and ovarian 164 cancer cell lines (Figure 3A-B). Similarly, C/EBP $\delta$ protein expression levels in chemotherapy naive 165 HGSC tissue by western blot analyses is low, consistent with immunohistochemical data of formalin 166 fixed tissue (Figure 3C). To further investigate the biological consequences of $\mathrm{C} / \mathrm{EBP} \delta$ expression in 167 cancer cell lines, we used three breast cancer cell lines (MCF7, T47D, MDA231), and an ovarian cancer 168 cell line (SKOV3). MCF7 and T47D have wildtype p53 whereas MDA231 and SKOV3 have p53 169 mutations $[\underline{41}, \underline{42}] . \mathrm{C} / \mathrm{EBP} \delta$ is expressed in estrogen receptor positive cancer cell lines, including MCF7 170 (highest), T47D and SKOV3 with no/low expression in estrogen receptor negative MDA-231 (Figure 171 3D). Similar to FTE cells, growth inhibitory effects of C/EBP $\delta$ overexpression were observed in cancer 172 cell lines which resulted in reduced cell growth in MCF7, T47D, SKOV3 but an increased growth in 173 MDA-231 compared to controls (Supplementary Figure 3A). Proliferation assays demonstrated that in 174 MCF7, C/EBP $\delta$ overexpression decreased cell growth at day 3 (3.6-Fold, p=0.01), day 5 (4.7-Fold, 
$175 \mathrm{p}=0.04)$, day $7(11.43$-Fold, $\mathrm{p}=0.002)$ and day 10 (30-Fold, $\mathrm{p}=0.002)$ compared to control cells

176 (Supplementary Figure 3B). A second breast cancer cell line, T47D, overexpressing C/EBP $\delta$

177 demonstrated a signifcanct decrease in cell proliferation at day $3(11.45$-Fold, $\mathrm{p}=0.00009)$, day 5 (11.67-

178 Fold, $\mathrm{p}=0.01)$ and day 10 (12.5-Fold, $\mathrm{p}=0.01)$ (Supplementary Figure 3B) but in the more mesenchymal

179 cell line, MDA231, C/EBP $\delta$ increased growth rates slightly compared to controls, albeit not statistically

180 significant $(\mathrm{p}=0.35)$ (Supplementary Figure 3A, 3B). Since C/EBP $\delta$ basal expression levels were low in

181 proliferating FTE and in HGSC, we used MCF7 cell line where basal levels of C/EBP $\delta$ can be clearly

182 detected by western blot analysis to determine if $\mathrm{C} / \mathrm{EBP} \delta$ is necessary to maintain proliferation (Figure

183 3D). A non-targeting (NT) control cell line and a CEBPD-knockdown (KD) stable cell line were

184 generated in MCF7 (Figure 3E). Loss of C/EBP $\delta$ expression in MCF7 had no significant effect on cell

185 growth (Figure $3 \mathrm{~F})$. However, $\mathrm{C} / \mathrm{EBP} \delta$ over-expression resulted in a decrease of anchorage independent

186 growth (1.6-fold, $\mathrm{p}=0.01$ ) (Figure 3G). In contrast, MDA231 with CEBPD overexpression resulted in

187 increased soft agar colony formation relative to control cells (Supplementary Figure 3C). In the context

188 of MCF7, a hormonally responsive breast cancer cell line, C/EBP $\delta$ expression is sufficient to inhibit

189 anoikis by anchorage independent growth.

191 C/EBP $\delta$ expression is associated with a MET and migratory potential in FTE and cancer

192 Epithelial to mesenchymal transition (EMT) has a fundamental role in cancer metastasis; restoration of 193 the mesenchymal to epithelial transition (MET) program should efficiently slow dissemination of tumor 194 cells [43]. Epithelial cells within fallopian tube undergo morphological changes during the menstrual 195 cycle evident under light microscopy [44, 45]. The FTE are characteristically pseudo-stratified epithelia 196 consisting of cuboidal and columnar epithelial cells of secretory and ciliated cells. During the pre197 ovulatory (follicular) phase there is an increase in proliferation [46]. At ovulation, the secretory cells 
reach peak activity and secrete their nutritive contents into the lumen of the tube, then reduce in height

199 to allow ciliated cells to move secretions by the beating of their cilia. Subsequently, in the post-

200 ovulatory (luteal) phase, both cell types reduce in height and there is partial deciliation [47]. C/EBP $\delta$

201 expression in the mouse ovary was previously reported to be mediated by the luteinizing hormone, LH

202 [48] and both mRNA and protein levels are higher in FTE in the luteal phase of the ovarian cycle [22].

203 Using a TMA of FTE $(\mathrm{n}=52)[\underline{22}, \underline{46}]$ annotated with BRCA1 mutation and ovarian cycle status, we

204 assessed expression of both vimentin and e-cadherin by IHC and image analysis (Figure 4A).

205 Concurrent TMA slides were stained for all three proteins. In general, C/EBP $\delta$ basal protein expression

206 was low. Interestingly, in the FTE luteal cases, where C/EBP $\delta$ expression was higher, we saw a

207 downward shift in vimentin ${ }^{+}$expressing FTE cells $(90.2 \%$ in the follicular phase to $68.5 \%$ in the luteal

208 phase, $\mathrm{p}=0.33)$. No significant changes in e-cadherin were observed $(91.0 \%$ in follicular phase to $88.9 \%$

209 in luteal phase, $\mathrm{p}=0.51$ ) (Figure 4A-B). In vitro, FTE with p53 mutations and hTERT retain a

210 mesenchymal phenotype which is characterized by vimentin expression as observed in FTE in vivo

211 (Figure 4D, Supplementary Figure 2B). FTE overexpressing C/EBP $\delta$ showed a distinctive epithelial-like

212 phenotype with well-defined sheets of adhering cells compared to control cells which had more

213 elongated mesenchymal shape (Figure 4C). Western blot analyses demonstrated a decrease in vimentin

214 and increase in e-cadherin expression in FTE overexpressing C/EBP $\delta$ compared to controls (Figure 4D,

215 Supplementary Figure 2B). This data suggested a role for C/EBP $\delta$ in regulating cellular phenotypes and

216 possibly cell differentiation. Overexpression of C/EBP $\delta$ in FTE significantly increased migration

217 compared to control cells (FTE57, 2.0-fold, p<0.01, and FTE19, 1.6-fold, p<0.01) (Figure 4E,

218 Supplementary Figure 2A), but did not affect migration in one cell line (Supplementary Figure 2E).

219 From our results, overexpression of $\mathrm{C} / \mathrm{EBP} \delta$ induces an MET phenotype by suppressing vimentin and 
220 increasing e-cadherin which resulted in an arrest of the cell cycle and decreased the migratory effects of 221 cells.

223 EMT genes such as SNAIL, TWIST and the ZEB family of transcription factors can change the 224 phenotypical characteristics of cells to regulate this phenomenon. E-cadherin expression has been 225 shown to be reduced in some primary ovarian carcinomas, and then re-expressed in ovarian carcinoma 226 effusions and at metastatic sites [49]. Further, ovarian carcinoma cells can co-express e-cadherin and the 227 EMT-associated n-cadherin suggesting that ovarian carcinoma cells undergo incomplete EMT [50, 51]. 228 We found similar co-expression of both e-cadherin and vimentin in benign FTE. Expression of e229 cadherin goes up in neoplastic STIC lesions while the EMT associated protein vimentin is decreased 230 (Supplementary Figure 7A-B). Furthermore, microarray analysis from a previous publication identified 231 the heterogenous expression of multiple EMT markers in normal fallopian tube epithelia across the 232 luteal and follicular phases (Supplementary Figure 7E). A panel of six HGSC probed for EMT/MET 233 markers using immunoblots demonstrated the variable expression levels of these markers, consistent 234 with other publications (Supplementary Figure 5C). IHC analysis of a STIC and HGSC sample showed 235 that vimentin and e-cadherin levels were low in HGSC, but e-cadherin levels were higher in STIC 236 relative to vimentin levels. (Supplementary Figure 7C, 7D). Quantitative RT-PCR (qPCR) showed a 237 significant increase in Snail, Twist and Zeb in FTE-CEBPD-OE cells compared to FTE-ctrls $(\mathrm{p}<0.01$, $238 \mathrm{n}=3$ ), in the 2 independent FTE cell lines (Figure 5A). In contrast, C/EBP $\delta$ overexpression resulted in a 239 decrease in protein expression levels of twist, slug, zeb1, vimentin and n-cadherin and an increase of 240 snail protein compared to control cells (Figure 5B) indicating C/EBP $\delta$ influences a MET program in 241 normal fallopian tube cells. 
243 In vitro, overexpression of C/EBP $\delta$ in SKOV3 led to an epithelial phenotype characterized by well-

244 defined cells compared to control cells, which had a more elongated, mesenchymal phenotype (Figure

245 6A, Supplementary Figure 4A). Furthermore, FACS analysis of SKOV3 cells demonstrated an increase 246 in $\mathrm{EpCAM}^{+}$cells and decrease in $\mathrm{Cd}_{49 \mathrm{f}^{+}}$relative to control (Supplementary Figure 4B). Across FTE 247 cells, $\mathrm{EPCAM}^{+}$cell expansion was noted, consistent with the cancer cell line (Supplementary Figure 248 4D). Similar to FTE, western blot analysis revealed that overexpression of C/EBP $\delta$ in SKOV3 induced 249 e-cadherin and decreased vimentin, zeb1 and zeb2 expression levels (Figure 6B). We then studied the 250 effect of C/EBP $\delta$ overexpression on breast cancer cells lines MCF7 and T47D which have higher overall 251 e-cadherin compared to the more mesenchymal MDA-231 cells. C/EBP $\delta$ protein expression levels are 252 highest in MCF7 associated with strong expression levels of epithelial markers, e-cadherin and CK8, 253 while MDA-231 has low C/EBP $\delta$ expression which is associated with low e-cadherin and CK8 254 expression and high vimentin expression (Figure 6C). EMT markers such as SNAIL and TWIST were 255 expressed in T47D cells and ZEB2 was expressed in all three cell lines (Supplementary Figure 5A). In $256 \mathrm{MCF7}$, overexpression of $\mathrm{C} / \mathrm{EBP} \delta$ resulted in an increase in e-cadherin expression while silencing of $257 \mathrm{C} / \mathrm{EBP} \delta$ reversed e-cadherin levels (Figure 6D). Using T47D cells overexpressing C/EBP $\delta$ there was no 258 effect on SNAIL and a slight decrease in TWIST expression (Supplementary Figure 5B). An in-vitro 259 observation of MCF7 cells with overexpression C/EBP $\delta$ using bright field microscopy showed cells 260 were more compact and rounded, indicative of an epithelial phenotype, compared to cells with a C/EBP $\delta$ 261 knockdown, which displayed filipodia and were less compact (Figure 6E). As in FTE, the 262 overexpression of $\mathrm{C} / \mathrm{EBP} \delta$ in $\mathrm{MCF} 7$ cells resulted in significantly more cells migrating relative to 263 control cells (1.74 fold, $\mathrm{p}=0.004)$ (Figure 6F). Taken together, as normal cells transition towards a 264 neoplastic state, $\mathrm{C} / \mathrm{EBP} \delta$ expression decreases resulting in less control over the cell cycle and as a 265 consequence pushes the cell to a more epithelial phenotype (MET) which in the context of high-grade 
serous cancer makes it more permissive to transformation (Figure 6G). In many in vitro and in vivo

267 models of EMT, polarized epithelial cells EMT, cells lose polarity, cell-cell adhesion and acquire

268 migratory and invasive properties [52]. In the FTE, a polarized epithelial layer of ciliated and secretory,

269 cytokeratin $18^{+}$cells, express both vimentin and e-cadherin and undergo a modified mesenchymal to 270 epithelial transition during serous tubal intraepithelial transformation as seen in Supplementary Figure 271 6A-B.

\section{C/EBPס induces an EMT regulatory phenotype}

274 It is well established that miRNAs regulate translation efficiency of genes by targeting mRNA. [53].

275 This can result in differences between the amount of protein and mRNA in a cell. Certain genes, such as $276 \mathrm{p} 53$, alter the expression of miRNA which results in changes in the phenotypical characteristics of cells. 277 Loss of p53 in mouse ovarian epithelial cells resulted in downregulation of miRNA-34b and miRNA$27834 \mathrm{c}$ which decreased proliferation and anchorage independent growth [54] . C/EBP $\delta$, itself has been 279 shown to be regulated by miRNA regulatory networks with IL6 and TNF[55]. To determine whether 280 there were differences in miRNA expression, the miRNA of FTE-CEBPD-OE was compared to FTE281 ctrl cells using a miRNA qPCR Array Human Ovarian cancer array (84 miRNAs). Comparisons were 282 made between FTE-19-CEBPD-OE/controls and FTE-57-CEBPD-OE/controls cell lines 283 (Supplementary Figure 2A). There were 10 miRNAs that were commonly differentially expressed 284 across both FTE-CEBPD over-expressing cells lines with a more than 2-fold change. Of these, let-7b285 5p, miR-125b-1-3p, miR-143-3p, miR-145-5p, miR-224-5p and miR-346 were up-regulated and miR286 26a-5p, miR-27a-3p, miR-106-5p, miR-200b-3 were down-regulated (Figure 5C, Supplementary Figure 287 2A). miR-200b was downregulated in cells where C/EBP $\delta$ was over-expressed. MiR-200b is 288 downregulated in ovarian cancer and has been known to target Zeb1, Zeb2 and Slug which are repressors 
of e-cadherin. [56, 57]. Low levels of miR-200 in addition to a decrease in e-cadherin results in an

290 increase in vimentin, n-cadherin, twist and snail [58]. Overexpression of leb-7b suppresses cancer cell

291 growth $[\underline{59}, \underline{60}]$ and therefore, may be one of the mechanisms by which C/EBP $\delta$ controls the fallopian

292 tube epithelial cell cycle. C/EBP $\delta$ has also been shown to be involved in miRNA gene networks with

293 IL6 - miR-26a-5p, TNF - miR-143-3p and miR-27a, CREBBP - miR-27b [55]. The results suggest a

294 role for $\mathrm{C} / \mathrm{EBP} \delta$ in altering the miRNA expression profile within normal FTE, suggesting a plausible

295 explanation for the discrepancy in mRNA and protein expression levels and the C/EBP $\delta$-MET induced

296 phenotype in the FTE cells. This data suggests a role for C/EBP $\delta$ in regulating EMT/MET in the context

297 of p53DN fallopian tube epithelia (Figure $6 \mathrm{H})$.

\section{Discussion}

300 In this study, we present a role for $\mathrm{C} / \mathrm{EBP} \delta$ in the regulation of an EMT/MET program during early 301 preneoplastic changes in the fallopian tube. Expression of $\mathrm{C} / \mathrm{EBP} \delta$ is high in $40 \%$ of STICs and expression from STIC to HGSC is maintained in the 40/60\% high-low ratio. High expression of C/EBP $\delta$ promotes a partial EMT/MET phenotype in FTE cells. C/EBP $\delta$ has a role in promoting migration of cells despite decreasing cell proliferation, a characteristic that has also been seen in mesenchymal-like breast cancer cells expressing YB-1 [61]. It is possible that CEBPD reduces proliferation rates thereby decreasing cell density allowing for the monolayer migration [62]. In the context of a p53 mutation and CEBPD, cells become more mesenchymal relative to baseline, which results in an MET phenotype. This

308 feature, as seen in the luteal phase of the ovarian cycle, results in slower growth. Independent of $309 \mathrm{C} / \mathrm{EBP} \delta$, the fallopian tube epithelial cells express a mesenchymal protein, vimentin, which changes 310 with ovulatory cycle. This highlights a new appreciation of the inherent epithelial and mesenchymal 311 features of FTE cells which either facilitate or inhibit tumorigenesis (Figure 7G,7H). 
313 Previously, by comparing hormonally driven changes of phenotypically normal FTE from women with

314 and without a BRCA1 mutation, we identified several differentially expressed genes with known

315 functions that promote tumor development and metastasis [22, 63]. Amongst these genes, C/EBP $\delta$, a 316 transcriptional regulator of cellular differentiation, inflammatory signaling, hypoxia adaptation and 317 metastatic progression, was increased during the luteal phase of the ovarian cycle [22]. C/EBP $\delta$ has 318 dualistic roles as a tumor suppressor or oncogene and has been called a master regulator gene. In breast 319 cancer cell line, MCF7, C/EBP $\delta$ was shown to decrease cylinD1 by mediating CCND1 degradation 320 through Cdc27/APC3 regulation $[\underline{64}, \underline{65}]$. In the epidermoid skin cancer cell line, A431, C/EBP $\delta$ also 321 decreased cancer cell proliferation induced by interacting with E2F1 and Rb [31]. In leukemia, CML, 322 KCL22 and K562 cell lines, expression of C/EBP $\delta$ was associated with downregulation of c-Myc and 323 cyclin $\mathrm{E}$ and upregulation of the cyclin-dependent kinase inhibitor p27 [28]. We sought to establish $324 \mathrm{C} / \mathrm{EBP} \delta$ 's role in the pathogenesis of high-grade serous ovarian cancer. Our tissue-based data revealed 325 that $77 \%$ of a cohort of HGSC cases had low C/EBP $\delta$ protein expression compared to LGSCs. This 326 difference may be reflective of the underlying pathogenic routes to these distinct tumors that arise in the 327 fallopian tube $[\underline{2}, \underline{66}$. HGSC is fast growing and has a high proliferative index compared to LGSC, 328 which is slow growing and indolent [67]. In our cohort of normal FTE and serous intraepithelial 329 carcinoma in situ (STIC) cases, C/EBP $\delta$ expression was inversely correlated to Ki67 expression. Higher 330 expression levels of $\mathrm{C} / \mathrm{EBP} \delta$ were associated with lower $\mathrm{Ki} 67$ staining and the trend was consistent in 331 HGSCs. This suggests that $\mathrm{C} / \mathrm{EBP} \delta$ is preferentially downregulated in highly proliferative tissues of the 332 fallopian tube and cancer. 
334 Since C/EBP $\delta$ expression is higher in the normal tissues compared to $70 \%$ of HGSC, we used an in vitro

335 FTE model to explore the effects of C/EBP $\delta$ overexpression in p53 dominant negative mutant cells [6].

336 In this study, C/EBP $\delta$ overexpression decreased cellular growth resulting in accumulation of the cells in

337 the G1/S phase of the cell cycle. This observation was extended to ovarian and breast cancer cell lines,

338 suggesting $\mathrm{C} / \mathrm{EBP} \delta$ is enough to inhibit growth. Unfortunately, endogenous levels of C/EBP $\delta$ were too

339 low to observe an additional decrease in endogenous protein levels in the modeled premalignant

340 fallopian tube cells. However, silencing C/EBP $\delta$ in MCF7 did not affect growth, suggesting that C/EBP $\delta$

341 is sufficient but not necessary for proliferation.

343 Within the normal tissue, $\mathrm{C} / \mathrm{EBP} \delta$ levels are higher in the luteal phase of the menstrual cycle relative to 344 the follicular phase. The luteal phase constitutes a highly inflammatory milieu with fallopian tube 345 epithelial cells undergoing differentiation to accommodate the motility of the ovum toward the ampulla 346 for impregnation. $\mathrm{C} / \mathrm{EBP} \delta$ is rapidly induced by inflammatory signals, cytotoxic factors and stressful 347 conditions, which are characteristics of the luteal phase $[\underline{23}, \underline{68]}$. Given that C/EBP $\delta$ has a dichotomous 348 role in regulating proliferation and differentiation [28], we hypothesized the expression of C/EBP $\delta$ 349 might be slowing the growth of cells to prepare them for a transitional state. Indeed, we describe $350 \mathrm{C} / \mathrm{EBP} \delta$ 's role for the first time in mediating an EMT/MET switch thereby providing FTE with 351 epithelial plasticity and enabling the migratory potential of these cells. We hypothesize higher levels of $352 \mathrm{C} / \mathrm{EBP} \delta$ in premalignant FTE might alter cell phenotype toward an epithelial fate with potential to 353 migrate to the ovarian surface epithelium.

355 One of the main points of debate regarding the fallopian tube epithelial origin of HGSC is how 356 mesenchymal cells of origin (i.e. fallopian tube epithelial cells) form serous epithelial ovarian 
carcinoma. Factors other than location and proximity contribute to seeding to the ovary by fallopian tube

358 precursor lesions. We hypothesized menstrual cycle associated genes with dual role of controlling

359 proliferation and differentiation might play an important role in this process. In our FTE cell culture

360 model, a p53 dominant negative $(\mathrm{R} 175 \mathrm{H})$ mutation was introduced to recapitulate one of the earliest

361 known events in HGSC, namely, the p53 signature. Loss of p53 was previously shown to induce

362 mesenchymal-like features in normal mammary epithelial cells [56]. We report similar findings in FTE.

363 We also observed expression of twist, slug, zeb1 and zeb2 at the protein level in p53 deficient FTE cells,

364 a feature consistent with a mesenchymal phenotype [69].

Here we showed C/EBP $\delta$ overexpression induced a partial MET, characterized by an increase in expression of epithelial markers, E-cadherin and CK7, and a decrease in expression of mesenchymal markers, including vimentin and n-cadherin. This was observed in FTE precursor lesions as well as cancer cell lines. Furthermore, upon $\mathrm{C} / \mathrm{EBP} \delta$ overexpression, a decrease in the expression of twist, slug and zeb1 were observed at the protein level. The data suggests a role for C/EBP $\delta$ in regressing the mesenchymal phenotype of p53 mutated FTE cells. The overexpression of C/EBP $\delta$ still increased the migratory rate of FTE cells and cancer cell lines. However, knocking down C/EBP $\delta$ in a cancer cell line did not demonstrate a significant difference in migration relative to controls. In urothelial cancers, $\mathrm{C} / \mathrm{EBP} \delta$ enhanced the invasiveness of UC cells through direct binding and upregulation of MMP2 [뭉. This highlights a dichotomous role of $\mathrm{C} / \mathrm{EBP} \delta$ as a tumor suppressor or oncogene with tumor promoting potentials depending on the tissue and the genomic context within which the gene is expressed. Primary fallopian tube cells have limited growth expansions in vitro []ㅡ and therefore, conducting these mutation along with $\mathrm{C} / \mathrm{EBP} \delta$ over-expression promotes cellular migration. 
381 Human Fallopian tube epithelial cells do not require intravasation to metastasize to the ovary and other

382 organs in the abdominal cavity. FTE cells are exposed to the peritoneal cavity where they can slough off 383 easily and disseminate to other locations. This study highlights new roles for C/EBP $\delta$ in HGSC and 384 separately features intriguing EMT/MET hybrid FTE phenotypes influenced by the ovulatory cycle. 385 This hybrid-pleiotropic phenotype in cancer is implicated in cancer metastasis, cancer stem cell 386 plasticity, chemoresistance and immune evasion [ $[\underline{11}, \underline{72}]$, all features also attributed to ovarian cancer 387 biology and heterogeneity. These EMT/MET hybrid protein expressions observed in the histologically 388 normal fallopian tube epithelia and pre-malignant lesions need further study to identify core 389 transcriptional and genomic/epigenomic networks driving fallopian tube epithelia transformation. 390 Further, FTE cells endogenously express well established mesenchymal markers like vimentin and 391 epithelial markers as e-cadherin simultaneously. Acquisition of mutant p53 is not required for these cells 392 to express mesenchymal genes but may play a role in promoting anoikis and survival in the peritoneal 393 cavity once detached from the basement membrane. During the ovulatory cycle, $\mathrm{C} / \mathrm{EBP} \delta$ decreases cell 394 growth and can influence mesenchymal gene expression potentially through miRNAs. Although the 395 connection between $\mathrm{C} / \mathrm{EBP} \delta$ and miRNA requires further study, several publications have highlighted 396 the role of C/EBP $\delta$ in regulating miRNA resulting in downstream phenotypical differences in cells with 397 and without $\mathrm{C} / \mathrm{EBP} \delta$, as is the case of miR-193b in urothelial human carcinoma's which demonstrated 398 and anti-tumorigenic function of C/EBP $\delta$ through miR-193b in NUTB1 human urothelial carcinoma cell 399 line [73]. In this context, we suggest a model whereby C/EBP $\delta$ 's initial expression in premalignant FTE 400 would slow cell cycle progression and allow these cells to acquire epithelial phenotype with potential to 401 avoid anoikis and migrate to the ovary. Once these cells have migrated to the ovary, decreased 402 expression of $\mathrm{C} / \mathrm{EBP} \delta$ promotes the proliferative potential of the cells which maintain a mesenchymal 
403 phenotype reminiscent of the cell of origin. Future studies are warranted to investigate which other 404 genes and/or gene networks promote these MET/EMT switches in early fallopian tube epithelial cells 405 transformation and whether these networks are elicited in chemoresistant ovarian cancer.

406

407

408

409

410

411

412

413

414

415

416

417

418

419

420

421

422

423

424

425 


\section{Material and Methods}

\section{Case collection}

428 The University Health Network Research Ethics Board approved the study protocol for collection of 429 tissue and clinical information for all patients. Each patient provided written informed consent allowing 430 for the collection and use of tissue for research purposes. H\&E sections of high-grade serous carcinoma, 431 borderline and low-grade serous carcinoma were reviewed by a gynecological pathologist (P.S.) prior to 432 use in the study. Diagnosis of each case was retrieved from the UHN ovarian tissue bank prior to review. 433 To validate immunohistochemical protein expression of samples, whole sections of tissue were cut from 434 formalin fixed paraffin embedded tissue and analyzed as in our previous publication [6, 13, 74]. A 435 previously published cohort $(n=15)$ of serous tubal intraepithelial carcinoma (STIC) cases using Abcam 436 morphological and immunohistological features including cellular crowding, loss of nuclear polarity and 437 presence of p53 and Ki67, from women having adnexal high-grade serous carcinoma [6, 13, 46].

\section{Immunohistochemistry}

440 Immunohistochemistry was performed using standard procedures as previously described [63] with the 441 following modifications. The following antibodies were used at these dilutions: Ki67 (Lab Vision, 442 Thermo Scientific, Waltham, Massachusetts, USA) 1/1000; p53 (Novocastra, Leica, Wetzlar, Germany)

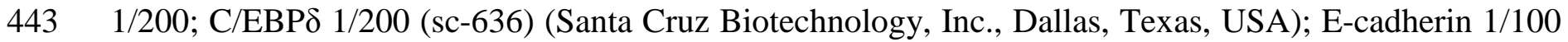
444 (AB15148) (Abcam, Cambridge, United Kingdom); Vimentin 1/100 (5741S) (Cell Signaling 445 Technology, Danvers, Massachusetts, USA). Appropriate negative and positive controls were performed 446 to determine specificity of antibodies. Stained slides were scanned using the ScanScope XT slide 447 scanner (Aperio Technologies, Inc, Leica) to create digital images at 40X magnification which were 448 then quantified for intensity and percentage of cells staining using a nuclear algorithm as previously 
449 described (Spectrum Plus, Image Analysis Toolbox, TMALab II, Aperio, Inc.) [22, 74]. Intensity levels 450 were based on an absorption range scale of 0-255 (0=black; 255=white). Weak Intensity Staining (1+) 451 ranged from 200-215; medium intensity staining (2+) from 180-200; and strong intensity staining (3+) 452 from $0-180$. Intensity levels +2 and +3 were combined to create a composite score for the percent 453 positive nuclei present in each case. Images were annotated to include only epithelium while excluding 454 stroma.

\section{Immunofluorescence}

457 Cells were grown in 6-well plates (Falcon) coated with collagen IV and fixed with $4 \%$ PFA for 5 458 minutes, permeabilized with $0.3 \%$ Triton-X/PBS then blocked with $5 \%$ goat serum (Gibco, Life 459 technologies) in PBS. Primary antibodies: C/EBPס (SC-636), CK18 (M701029) (Dako, Agilent 460 Technologies, Santa Clara, California, United States), Pax8 (10336-1-AP) (ProteinTech, Rosemont, IL, 461 USA), E-cadherin (AB15148) (Abcam), Vimentin (5741S) (Cell Signaling), were applied overnight at $4624{ }^{\circ} \mathrm{C}$. Primary antibody was removed by washing samples with 1x PBS three times and was incubated 463 with appropriate fluorophore-labeled secondary antibodies (Jackson ImmunoResearch Laboratories, 464 West Grove, PA, USA) and Vectashield (H-1200, Vector Laboratories, Burlingame, CA, USA) in a dark 465 area for $45 \mathrm{~min}$. Cells were again washed for three times with 1x PBS and was mounted onto a coverslip 466 and dried in the dark for $10 \mathrm{~min}$. Samples were visualized using a Leica Axioimager (Leica).

469 Surgical samples were obtained from the University Health Network with patient consent and Research 470 Board Ethics Approval. In brief, fimbriae were collected after prophylactic hysterectomy or salpingo471 oophorectomy and incubated for 4-16 hours at $37^{\circ} \mathrm{C}$ in pronase and subsequently cultured as previously 
472 described [6]. Cells were immortalized via infection with a lentiviral dominant negative TP53 (R175H)

473 vector and retroviruses human telomerase (hTERT) [6]. Cells were infected with a lenti-viral C/EBP $\delta$ 474 overexpression construct 24 to 48 hours after cells reached $70 \%$ confluence. Each plate was grown till 475 confluence and harvested for different molecular assays. For biological replicates, all molecular analyses 476 of cell lines for RNA and protein extraction were performed from the cell population. That is, at time of 477 collection, cells were divided into two pellets. Additionally, for FACS and immunofluorescence, the same population of cells were pelleted for RNA and protein assays.

\section{Cell lines}

481 SKOV3, OVCAR3, MCF7, MDA231, T47D were obtained from the ATCC (Manassa, Virginia, USA). 482 SKOV3 (ATCC-HBT-77) was grown in McCOY's 5a (Life technologies, Carlsbad, California, USA) 483 supplemented with $10 \%$ fetal bovine serum (FBS, Wisent technologies, St-Bruno, Quebec 484 Canada). MCF7 (ATCC-HTB-22) and MDA231 (ATCC-HTB-26) cells were grown in DMEM/F12 485 (Life technologies), supplemented with 10\% FBS. T47D (ATCC-HTB-133) and OVCAR3 (HTB-161) 486 cells were grown in RPMI 1640 (Life technologies), supplemented with 10\% and 20\% FBS, 487 respectively. Culture method was followed as described by ATCC for each cell line.

\section{Virus Production and Infection}

490 HEK-293T cells were seeded at a density of $1.0 \times 10^{\wedge} 6$ cells per plate in a $6 \mathrm{~cm}$ tissue culture dish 491 overnight with low antibiotic growth media (DMEM + 10\% FBS). Cells were incubated until 70\% 492 confluence was achieved. A mixture of 3 transfection plasmids were produced by combining $2 \mu \mathrm{g}$ 493 pMDG.2 (Addgene \#12259) (Addgene, Watertown, Massachusetts, USA); $4 \mu \mathrm{g}$ of pCMV delta R8.2 494 (Addgene \#12263) and $5 \mu \mathrm{g}$ of vector (pCDH-CMV-MCS-EF1-GFP empty, pCDH-CMV-MCS-EF1- 
GFP-CEBPD - CEBPD-OE). As per manufacturer's protocol, GenJet DNA In-vitro Transfection reagent

496 (Ver. II) (SignaGen Laboratories, Rockville, MD, USA) was used as the transfection reagent. Reagents

497 were added to each plate drop-wise and was incubated overnight (16 hours, $37^{\circ} \mathrm{C}, 5 \% \mathrm{CO}_{2}$ ). $\mathrm{Next}$ day, 498 media was subsequently removed and replaced with high BSA growth media and again incubated for 24 499 hrs. Supernatant was then collected and passed through a 0.45 um filter and snap frozen and stored at $50080^{\circ} \mathrm{C}$ until required for use. A second round of collection was performed 72 hours after virus 501 transfection and was also filtered and stored at $-80^{\circ} \mathrm{C}$.

503 Protein isolation, western blot and antibodies

504 Cell samples were washed with 1x PBS and trypsinized using 0.25\% trypsin dissociation reagent (Life 505 technologies) for $5 \mathrm{~min}$ at 37 degree Celsius. TNS was added to samples and was spun at $1000 \mathrm{rpm}$ for 5 506 minutes. Supernatant was aspirated, and samples were placed on ice before lysing. Cells were lysed with 507 NP40 buffer (Life technologies) and protease inhibitor cocktail (Thermo Scientific). Samples were 508 incubated for $15 \mathrm{~min}$ on ice and subsequently clarified by centrifugation (14000rpm for 30min). The 509 supernatant was collected and quantified using BioRad DC Protein Assay (BioRad Laboratories, 510 Hercules, California, United States). Samples were re-suspended in 4x LDS sample buffer (Life 511 technologies), boiled and 30ug of protein was resolved by SDS-Page 4-12\% Bis-tris Gels (Novex, Life 512 technologies). Protein samples were transferred to BioRad PVDF membrane and were blocked with 5\% 513 milk powder dissolved in 1x TBS-T for one hour or overnight. Primary antibodies were diluted in 514 blocking buffer (5\% skimmed milk, Nestle, Vevey, Switzerland) and incubated with membranes 515 overnight at $4^{\circ} \mathrm{C}$. Membranes were washed for $10 \mathrm{~min}$, three times and probed with secondary antibody, 516 washed three times for $15 \mathrm{~min}$ using $1 \mathrm{x}$ TBS-T and developed using ECL Prime western blotting 517 detection reagent (GE Healthcare Life Sciences, Pittsburgh, PA, USA). Each membrane was imaged 
518 using BioRad ChemiDoc and images analyzed using BioRad Image Lab software (BioRad). The

519 following antibodies were used in western blots: C/EBP $\delta$ (Santa Cruz, SC-636, 1/200), B-actin (Sigma, 520 A2228, 1:1000) (Sigma-Aldrich, St. Louis, Missouri, USA), P53 (Santa Cruz; sc-126, 1/500), PAX8 521 (ProteinTech, 10336-1-AP, 1/1000), Snail1 (Cell Signaling, 3895S, 1/500), Slug (Cell Signaling, 9585S, 522 1/500) Vimentin (Cell Signaling, 5741S, 1/500), E-cadherin (Abcam, AB15148 1/500), N-cadherin 523 (SantaCruz, sc-7939, 1/500), SIP1 (Santa Cruz, sc-48789, 1/200), Twist (Santa cruz, sc-81417, 1/500) 524 and goat HRP-IgG (anti-mouse or anti-rabbit) (Santa Cruz, 1:10,000).

\section{Cloning Strategy}

527 Overexpression vectors were constructed using pCDH-CMV-MCS-EF1-GFP cloning/ expression vector 528 (System Biosciences, Palo Alto, CA,USA). C/EBPS insert was cut out of a PCR4 TOPO cloning vector 529 (Invitrogen, Carlsbad, California, USA) using EcoRI (New England Biolabs (NEB), Ipswich, 530 Massachusetts, USA). The cDNA was then ligated into pCDH-CMV-MCS-EF1-GFP using Takara 531 ligation Kit (Clontech, Mountain View, California, USA) and was confirmed in the expression vector by 532 DNA sequencing at The Centre for Applied Genomics (SickKids, Toronto, Ontario, Canada). Lentivirus 533 was produced using HEK-293T cells (Clontech) and the overexpression vector was confirmed in the 534 cells by GFP fluorescence and western blot (Figure 2A-B, Supplementary Figure 4A).

\section{Fluorescence Activated Cell Sorting (FACS)}

537 For cell cycle regulation assessment, cells were washed in 1x PBS and 10uM BrdU-APC was added to 538 cells in a dark environment. Plates with BrdU were then incubated at 37 degrees for 4 hrs. Cells were 539 then washed twice with ice cold PBS, trypsinized with $0.25 \%$ trypsin dissociation reagent and 540 neutralized for counting. Nuclei preparation and staining was performed by adding $0.08 \%$ Pepsin and 
$5412 \mathrm{~N} \mathrm{HCl}$ and IFA/0.5\% Tween20 (Sigma). Samples were incubated in the dark ad 100ul anti-BrdU-APC

542 was added while samples were incubated on ice. $5 \mathrm{ug} / \mathrm{ml}$ of propidium iodide was added to cells and

543 incubated on ice for $15 \mathrm{~min}$. Flow cytometry was carried out on BD FACS Calibur (BD biosciences, San 544 Jose, CA, USA). Data was analyzed using FlowJo v10 (Flowjo LLC, Ashland, OR, USA). Cell surface 545 staining was performed on single cell suspension. EpCAM-PE (Life technologies, VU-1D9) and CD49f546 APC (FAB13501A) (R\&D Biosystems, Minneapolis, Minnesota, USA) was added to the cell suspension 547 and incubated in the dark for 40-60min. Cells were then washed and centrifuged at $400 \mathrm{~g}$ for $5 \mathrm{~min}$ at 548 room temperature and subsequently vortexed to dissociate pellet. Stained cells were re-suspended in 549 staining buffer and flow cytometry acquired on BD Calibur flow cytometer (BD biosciences).

\section{Soft Agar Assay}

552 Base agar (1\% agarose Difco Agar Noble, BD biosciences ) was added to 6 well plates and allowed to 553 solidify for $5 \mathrm{~min}$. Top agarose layer was made by combining $0.7 \%$ agarose with media. 5000 cells were 554 added to the mixture and plated on 6 well plate. Cells were kept in an incubator at $37^{\circ} \mathrm{C}$ for 14 days. 555 Cells were fed twice a week. After 14 days, each plate well was stained with $0.5 \mathrm{ml}$ of crystal violet $556(0.005 \%)$ for approximately $1 \mathrm{hr}$. Plates were then imaged using a dissecting microscope and camera 557 setup (Leica). Images were imported into Image J (National Institute of Health, Bethesda, Maryland, 558 USA) and analyzed.

\section{Quantitative PCR}

561 RNA was isolated from FTE cells lysed with Trizol reagent (Invitrogen). 1ug of total RNA was reverse562 transcribed using qScript cDNA SuperMix (Quanta Biosciences, Beverly, MA, USA). Real-time 563 quantitative PCR (RT-qPCR) was performed using PerfeCTa Sybr Green FastMix, Rox (Quanta 
564 Biosciences) according to the manufacturer's protocol using the ABI PRISM 7900HT Sequence

565 Detection System (Applied Biosystems, Foster City, California, USA). The target CT values were

566 normalized to b-actin. The $\mathrm{N}$-fold differential expression was assessed using the 2- ${ }^{(\Delta \Delta \mathrm{CT})}$ method to 567 determine differences between treated cells and controls. Primer sequences were obtained from 568 PrimerBank [75, 76]. qRT-PCR as mean \pm s.e.m. Student's t-test with $\mathrm{n}=3, \mathrm{p} \leq 0.05$ unless noted 569 otherwise in text. $\mathrm{N}=3$ represents 3 independent experiments with 3 technical replicates within each 570 experiment.

\section{miRNA Assay}

573 FTE cells were harvested from $6 \mathrm{~cm}$ plates and RNA was isolated using miRNAeasy micro kit (Qiagen, 574 Hilden, Germany). Quantity and quality of total RNA was analyzed using Nanodrop (Thermo 575 Scientific). RNA was reverse transcribed into cDNA using miScript II RT kit (Qiagen). cDNA was then 576 processed using miScript SYBR Green PCR kit (Qiagen) and was run on miScript miRNA PCR Array 577 Human Ovarian Cancer plates (Qiagen, MIHS-110ZE-4, 384 well plate). PCR plates were read the ABI 578 PRISM 7900HT Sequence Detection System (Applied Biosystems). Results were outputted with 2- $\Delta$ Ct 579 values for each gene in each treatment group compared to the control group $(n=3)$. Qiagen software was 580 used to analyze results and student's t-test provided statistically significant miRNA $(\mathrm{p}<0.05)$.

Wound-healing Assay

58363857 p53DN-hTERT, 3619 p53DN-hTERT cells were seeded in 96-well plates (Essen ImageLock) and 584 grown to confluence. Scratch wounds were generated using the 96-pin WoundMaker (Essen BioScience, 585 Ann Arbor, MI, USA) and wells were gently washed with 1xPBS to remove non-adherent cells. Cells 
were imaged using the INCUCYTETM Kinetic Imaging System (Essen BioScience) and wound width

587 was determined using the INCUCYTE ${ }^{\mathrm{TM}}$ cell migration software module.

589 Migration Assay

590 MCF7 cells and FTE cells were seeded onto Costar Transwell migration assay (Corning Costar 3472)

591 (Corning Inc., Corning, New York, USA) at a density of 50000 cells. Plated cells were initially grown in 592 serum free media for 24 hrs. After trypsinization using Trypsin (EDTA 00.25\%) (Life Technologies), 593 cells were counted and plated onto the Transwell migration assay which was then placed in DMEM/F12 $594+10 \%$ FBS for 12 hours. Transwell's were then removed from assay and washed PBS and H2O to 595 remove unbound cells. $1 \%$ Crystal Violet $+2 \%$ ethanol was added to the Transwell and allowed to 596 incubate at room temperature for 15 minutes at which point the wells were rinsed with $\mathrm{H} 2 \mathrm{O}$ and dried. 597 Each well was imaged using an inverted microscope (Leica) and cells were counted in ImageJ (Image J 598 labs). Results were analyzed and graphed using GraphPad graphing software (GraphPad, La Jolla 599 California USA)

600

\section{Proliferation Assay}

602 For Proliferation Assay, 3.0 X $10^{4}$ cells were seeded onto a 6- well plate (Falcon, Corning Inc.) and 603 plated with $2 \mathrm{ml}$ of fresh media. Cells were counted every 1-3 days using CyQuant Direct Proliferation 604 Assay kit (Life technologies). An inverted plate reader (Flexstation 3) (Molecular Devices, San Jose, 605 California, USA) was used to quantify the amount of fluorescence emitted by live cells and provide a 606 count of live cells present. Softmax Pro software (Molecular Devices) was used to analyze the results. 
610 Statistical analysis was performed using GraphPad Prism Software (GraphPad). The Log-rank test was 611 used in Kaplan-Meir, Mantel-Cox regression analysis to compare C/EBP $\delta$ expression on overall survival 612 and progression free survival. MicroRNA analysis was performed using Qiagen SaBiosciences miScript 613 miRNA PCR Array Data Analysis Software (Qiagen). Values are expressed as means \pm SEM and were 614 performed three times, t-tests were performed to determine statistical significance, unless otherwise noted. A value with $\mathrm{p}<0.05$ was considered to be statistically significant.

616 and SG; Writing, review and editing: all authors.

\section{Conflict of Interest}

630 The authors declare no competing interests.

\section{Supplementary Information is available}




\section{Reference:}

634 1. Bowtell, D.D., et al., Rethinking ovarian cancer II: reducing mortality from high-grade serous ovarian cancer. Nat Rev Cancer, 2015. 15(11): p. 668-79.

2. Vang, R., M. Shih Ie, and R.J. Kurman, Fallopian tube precursors of ovarian low-and highgrade serous neoplasms. Histopathology, 2013. 62(1): p. 44-58.

3. Piek, J.M., et al., Women harboring BRCA1/2 germline mutations are at risk for breast and female adnexal carcinoma. Int J Gynecol Pathol, 2003. 22(3): p. 315-6; author reply 315-6.

4. Piek, J.M.J., et al., Dysplastic changes in prophylactically removed Fallopian tubes of women predisposed to developing ovarian cancer. The Journal of Pathology, 2001. 195(4): p. 451-456.

5. $\quad$ Folkins, A.K., et al., A candidate precursor to pelvic serous cancer (p53 signature) and its prevalence in ovaries and fallopian tubes from women with BRCA mutations. Gynecol Oncol, 2008. 109(2): p. 168-73.

6. George, S.H., et al., Loss of LKB1 and p53 synergizes to alter fallopian tube epithelial phenotype and high-grade serous tumorigenesis. Oncogene, 2015.

7. George, S.H. and P. Shaw, BRCA and Early Events in the Development of Serous Ovarian Cancer. Front Oncol, 2014. 4: p. 5.

8. Karst, A.M., K. Levanon, and R. Drapkin, Modeling high-grade serous ovarian carcinogenesis from the fallopian tube. Proceedings of the National Academy of Sciences of the United States of America, 2011. 108(18): p. 7547-52.

9. Jazaeri, A.A., et al., Molecular requirements for transformation of fallopian tube epithelial cells into serous carcinoma. Neoplasia, 2011. 13(10): p. 899-911.

10. Sehdev, A.S., et al., Serous tubal intraepithelial carcinoma upregulates markers associated with high-grade serous carcinomas including Rsf-1 (HBXAP), cyclin E and fatty acid synthase. Mod Pathol, 2010. 23(6): p. 844-855.

11. Visvanathan, K., et al., Diagnosis of serous tubal intraepithelial carcinoma based on morphologic and immunohistochemical features: a reproducibility study. The American journal of surgical pathology, 2011. 35(12): p. 1766-75.

12. Shaw, P.A., et al., Candidate serous cancer precursors in fallopian tube epithelium of BRCA1/2 mutation carriers. Modern Pathology, 2009. 22(9): p. 1133-1138.

13. Milea, A., et al., Retinoblastoma pathway deregulatory mechanisms determine clinical outcome in high-grade serous ovarian carcinoma. Mod Pathol, 2013.

14. Levanon, K., et al., FOXO3a loss is a frequent early event in high-grade pelvic serous carcinogenesis. Oncogene, 2014. 33(35): p. 4424-32.

15. Norquist, B.M., et al., The molecular pathogenesis of hereditary ovarian carcinoma: alterations in the tubal epithelium of women with BRCA1 and BRCA2 mutations. Cancer, 2010. 116(22): p. 5261-71.

16. Cancer Genome Atlas, N., Integrated genomic analyses of ovarian carcinoma. Nature, 2011. 474(7353): p. 609-15.

17. Karst, A.M., et al., Cyclin el deregulation occurs early in secretory cell transformation to promote formation of fallopian tube-derived high-grade serous ovarian cancers. Cancer Res, 2014. 74(4): p. 1141-52.

18. Karst, A.M., et al., Stathmin 1, a marker of PI3K pathway activation and regulator of microtubule dynamics, is expressed in early pelvic serous carcinomas. Gynecologic oncology, 2011. 123(1): p. 5-12. 
19. Kuhn, E., et al., Telomere length in different histologic types of ovarian carcinoma with emphasis on clear cell carcinoma. Mod Pathol, 2011. 24(8): p. 1139-45.

20. $\quad$ Lengyel, E., Ovarian cancer development and metastasis. Am J Pathol, 2010. 177(3): p. 105364.

21. Sodek, K.L., et al., Cell-cell and cell-matrix dynamics in intraperitoneal cancer metastasis. Cancer Metastasis Rev, 2012. 31(1-2): p. 397-414.

22. George, S.H., et al., Identification of abrogated pathways in fallopian tube epithelium from BRCA1 mutation carriers. The Journal of pathology, 2011. 225(1): p. 106-17.

23. Ramji DP, F.P., CCAAT/enhancer-binding proteins: structure, function and regulation. Biochem J., 2002. 365(Pt 3): p. 561-75.

24. Sterneck, K.B.a.E., The Many Faces of C/EBPS and their Relevance for Inflammation and Cancer. Int J Biol Sci., 2013. 9(9): p. 917-933.

25. Wu, S.-R., et al., CCAAT/Enhancer-binding Protein $\delta$ Mediates Tumor Necrosis Factor $\alpha$ induced Aurora Kinase C Transcription and Promotes Genomic Instability. The Journal of Biological Chemistry, 2011. 286(33): p. 28662-28670.

26. Carro, M.S., et al., The transcriptional network for mesenchymal transformation of brain tumours. Nature, 2010. 463(7279): p. 318-25.

27. Cooper, L.A.D., et al., The Tumor Microenvironment Strongly Impacts Master Transcriptional Regulators and Gene Expression Class of Glioblastoma. The American Journal of Pathology, 2012. 180(5): p. 2108-2119.

28. Gery, S., et al., C/EBPS expression in a BCR-ABL-positive cell line induces growth arrest and myeloid differentiation. Oncogene, 2005. 24: p. 1589.

29. Sanford, D.C. and J.W. DeWille, C/EBPdelta is a downstream mediator of IL-6 induced growth inhibition of prostate cancer cells. Prostate, 2005. 63.

30. Hutt, J.A. and J.W. DeWille, Oncostatin M induces growth arrest of mammary epithelium via a CCAAT/enhancer-binding protein delta-dependent pathway. Mol Cancer Ther, 2002. 1.

31. Pan, Y.-C., et al., CEBPD reverses RB/E2F1-mediated gene repression and participates in HMDB-induced apoptosis of cancer cells. Clinical Cancer Research, 2010.

32. Ko, C.-Y., et al., Epigenetic Silencing of CCAAT/Enhancer-binding Protein $\delta$ Activity by YY1/Polycomb Group/DNA Methyltransferase Complex. Journal of Biological Chemistry, 2008. 283(45): p. 30919-30932.

33. Tang, D., G.S. Sivko, and J.W. DeWille, Promoter methylation reduces C/EBPS (CEBPD) gene expression in the SUM-52PE human breast cancer cell line and in primary breast tumors. Breast Cancer Research and Treatment, 2006. 95(2): p. 161-170.

34. Takayuki Ikezoe, S.G., Dong Yin, James O'Kelly, Lise Binderup, Nathan Lemp, Hirokuni Taguchi and H. Phillip Koeffler, CCAAT/Enhancer-Binding Protein $\delta:$ A Molecular Target of 1,25-Dihydroxyvitamin D3 in Androgen-Responsive Prostate Cancer LNCaP Cells. Cancer Res, 2005. 65(11): p. 4762-4768.

35. Kuppusamy Balamurugan, S.S., Kimberly D. Klarmann, Youhong Zhang, Vincenzo Coppola, Glenn H. Summers, Thierry Roger, Deborah K. Morrison, Jonathan R. Keller \& Esta Sterneck, $F B X W 7 \alpha$ attenuates inflammatory signalling by downregulating $C / E B P \delta$ and its target gene Tlr4. Nature Communications, 2013. 4(1662): p. 1-12.

36. Gigliotti, A.P., et al., Nulliparous CCAAT/enhancer binding protein delta (C/EBPdelta) knockout mice exhibit mammary gland ductal hyperlasia. Exp Biol Med, 2003. 228. 
37. Thangaraju M, R.M., Bierie B, Raffeld M, Sharan S, Hennighausen L, Huang AM, Sterneck E., C/EBPdelta is a crucial regulator of pro-apoptotic gene expression during mammary gland involution. Development, 2005. 132(21): p. 4675-85.

38. O'Rourke, J., R. Yuan, and J. DeWille, CCAAT/enhancer-binding protein-delta (C/EBP-delta) is induced in growth-arrested mouse mammary epithelial cells. J Biol Chem, 1999. 272.

39. Hutt, J.A., J.P. O'Rourke, and J. DeWille, Signal transducer and activator of transcription 3 activates CCAAT enhancer-binding protein delta gene transcription in GO growth-arrested mouse mammary epithelial cells and in involuting mouse mammary gland. J Biol Chem, 2000. 275.

40. Medrano, M., et al., Interrogation of Functional Cell-Surface Markers Identifies CD151 Dependency in High-Grade Serous Ovarian Cancer. Cell Rep, 2017. 18(10): p. 2343-2358.

41. Connor, P.M., et al., Characterization of the \&Tumor Suppressor Pathway in Cell Lines of the National Cancer Institute Anticancer Drug Screen and Correlations with the Growth-Inhibitory Potency of 123 Anticancer Agents. Cancer Research, 1997. 57(19): p. 4285.

42. Ince, T.A., et al., Characterization of twenty-five ovarian tumour cell lines that phenocopy primary tumours. Nat Commun, 2015. 6: p. 7419.

43. Thiery, J.P., et al., Epithelial-Mesenchymal Transitions in Development and Disease. Cell, 2009. 139(5): p. 871-890.

44. Donnez, J., et al., Cyclic changes in ciliation, cell height, and mitotic activity in human tubal epithelium during reproductive life. Fertility and sterility, 1985. 43(4): p. 554-9.

45. Julie Crow, N.N.A., Jackie Lewin,Robert W. Shaw, Physiology: Morphology and ultrastructure of Fallopian tube epithelium at different stages of the menstrual cycle and menopause. Human Reproduction 1994. 9(12): p. 2224-2233.

46. George, S.H., A. Milea, and P.A. Shaw, Proliferation in the normal FTE is a hallmark of the follicular phase, not BRCA mutation status. Clinical cancer research : an official journal of the American Association for Cancer Research, 2012. 18(22): p. 6199-207.

47. Patek, E., L. Nilsson, and E. Johannisson, Scanning Electron Microscopic Study of the Human Fallopian Tube. Report II. Fetal Life, Reproductive Life, and Postmenopause**Supported by Swedish Medical Research Council Grant B72-12x-2712-04, Karolinska Institute, Stockholm, the Swedish International Development Authority, Stockholm, the Ford Foundation, U.S.A., and JEOL Inc., Tokyo, Japan. Fertility and Sterility, 1972. 23(10): p. 719-733.

48. Huang, A.-M., et al., The Cebpd (C/EBPd) Gene Is Induced by Luteinizing Hormones in Ovarian Theca and Interstitial Cells But Is Not Essential for Mouse Ovary Function. PLoS One, 2007(12): p. e1334-1341.

49. Davidson, B., et al., E-cadherin and $\alpha$-, $\beta$-, and $\gamma$-catenin protein expression is up-regulated in ovarian carcinoma cells in serous effusions. The Journal of Pathology, 2000. 192(4): p. 460-469.

50. ElMoneim, H.M.A. and N.M. Zaghloul, Expression of E-cadherin, $N$-cadherin and snail and their correlation with clinicopathological variants: an immunohistochemical study of 132 invasive ductal breast carcinomas in Egypt. Clinics (Sao Paulo, Brazil), 2011. 66(10): p. 17651771.

51. Davidson, B., C.G. Tropé, and R. Reich, Epithelial-mesenchymal transition in ovarian carcinoma. Frontiers in oncology, 2012. 2: p. 33-33.

52. Kalluri, R. and R.A. Weinberg, The basics of epithelial-mesenchymal transition. The Journal of clinical investigation, 2009. 119(6): p. 1420-8.

53. Trobaugh, D.W. and W.B. Klimstra, MicroRNA Regulation of RNA Virus Replication and Pathogenesis. Trends in Molecular Medicine, 2017. 23(1): p. 80-93. 
54. Corney, D.C., et al., \&lt;em\&gt;MicroRNA-34b\&lt;/em\&gt; and \&lt;em\&gt;MicroRNA$34 c \& l t ; /$ em\&gt; Are Targets of p53 and Cooperate in Control of Cell Proliferation and Adhesion-Independent Growth. Cancer Research, 2007. 67(18): p. 8433.

55. Anandaram, H. and D.A. Anand, Computational analysis of micro RNAs compatibility in pharmacogenomic based regulatory networks of psoriatic arthritis: An initiation towards identifying a potential miRNA to treat psoriatic arthritis. Biocatalysis and Agricultural Biotechnology, 2018. 16: p. 545-547.

56. Chang, C.-J., et al., p53 regulates epithelial-mesenchymal transition and stem cell properties through modulating miRNAs. Nature Cell Biology, 2011. 13: p. 317+.

57. Li, Y., et al., Up-regulation of miR-200 and let-7 by natural agents leads to the reversal of epithelial-mesenchymal transition in gemcitabine-resistant pancreatic cancer cells. Cancer research, 2009. 69(16): p. 6704-6712.

58. Bilyk, O., et al., Epithelial-to-Mesenchymal Transition in the Female Reproductive Tract: From Normal Functioning to Disease Pathology. Frontiers in Oncology, 2017. 7: p. 145.

59. $\mathrm{Xu}, \mathrm{H}$., et al., Let-7b-5p regulates proliferation and apoptosis in multiple myeloma by targeting IGF1R. Acta Biochim Biophys Sin (Shanghai), 2014. 46(11): p. 965-72.

60. Yu, J., et al., Let-7b inhibits cell proliferation, migration, and invasion through targeting Cthrc1 in gastric cancer. Tumour Biol, 2015. 36(5): p. 3221-9.

61. Tognon, C., T. Ng, and P.H.B. Sorensen, Reduced proliferation and enhanced migration: Two sides of the same coin? Molecular mechanisms of metastatic progression by YB-1 AU Evdokimova, Valentina. Cell Cycle, 2009. 8(18): p. 2901-2906.

62. Tlili, S., et al., Collective cell migration without proliferation: density determines cell velocity and wave velocity. Royal Society Open Science, 2018. 5(5): p. 172421.

63. Tone, A.A., et al., Gene Expression Profiles of Luteal Phase Fallopian Tube Epithelium from BRCA Mutation Carriers Resemble High-Grade Serous Carcinoma. Clinical Cancer Research, 2008. 14(13): p. 4067-4078.

64. Ikezoe, T., et al., CCAAT/enhancer-binding protein delta: a molecular target of 1,25dihydroxyvitamin D3 in androgen-responsive prostate cancer LNCaP cells. Cancer Res, 2005. 65.

65. Pawar, S.A., et al., C/EBP \{delta\} targets cyclin D1 for proteasome-mediated degradation via induction of CDC27/APC3 expression. Proc Natl Acad Sci U S A, 2010. 107(20): p. 9210-5.

66. Nik, N.N., et al., Origin and pathogenesis of pelvic (ovarian, tubal, and primary peritoneal) serous carcinoma. Annu Rev Pathol, 2014. 9: p. 27-45.

67. Vang, R., M. Shih Ie, and R.J. Kurman, Ovarian low-grade and high-grade serous carcinoma: pathogenesis, clinicopathologic and molecular biologic features, and diagnostic problems. Adv Anat Pathol, 2009. 16(5): p. 267-82.

68. Yamaguchi, J., et al., Inflammation and hypoxia linked to renal injury by CCAAT/enhancerbinding protein $\delta$. Kidney International, 2015. 88(2): p. 262-275.

69. Kogan-Sakin, I., et al., Mutant p53(R175H) upregulates Twist1 expression and promotes epithelial-mesenchymal transition in immortalized prostate cells. Cell Death and Differentiation, 2011. 18(2): p. 271-281.

70. Wang, Y.-H., et al., CEBPD amplification and overexpression in urothelial carcinoma: a driver of tumor metastasis indicating adverse prognosis. Oncotarget, 2015. 6(31): p. 31069-31084.

71. Mittal, V., Epithelial Mesenchymal Transition in Tumor Metastasis. Annual Review of Pathology: Mechanisms of Disease, 2018. 13(1): p. 395-412. 
812 72. Title, A.C., et al., Genetic dissection of the miR-200-Zeb1 axis reveals its importance in tumor 813 differentiation and invasion. Nature Communications, 2018. 9(1): p. 4671.

814 73. Lin, S.-R., et al., MiR-193b Mediates CEBPD-Induced Cisplatin Sensitization Through 815 Targeting ETS1 and Cyclin D1 in Human Urothelial Carcinoma Cells. Journal of Cellular 816 Biochemistry, 2017. 118(6): p. 1563-1573.

817 74. May, T., et al., Low malignant potential tumors with micropapillary features are molecularly 818 similar to low-grade serous carcinoma of the ovary. Gynecol Oncol, 2010. 117(1): p. 9-17.

819 75. Spandidos, A., et al., PrimerBank: a resource of human and mouse PCR primer pairs for gene expression detection and quantification. Nucleic Acids Res, 2010. 38(Database issue): p. D7929.

76. Wang, X. and B. Seed, A PCR primer bank for quantitative gene expression analysis. Nucleic Acids Res, 2003. 31(24): p. e154. 


\section{Figure Legends}

842 Figure 1. C/EBP $\delta$ is differentially expressed between serous histotypes. A. Representative IHC 843 staining of $\mathrm{C} / \mathrm{EBP} \delta$ showing examples of HGSC and LGSC expressing low and high $\mathrm{C} / \mathrm{EBP} \delta$ 844 expression, respectively. Percentage of $\mathrm{C} / \mathrm{EBP} \delta$ staining was scored from 0 (low) to 3 (high). B. 845 Histogram shows C/EBP $\delta$ expression levels stratified by scores across a cohort of HGSC (N=366) and 846 LGSC $(\mathrm{N}=26)$. C. Dot plot of representative samples shows decreasing C/EBP $\delta$ IHC staining across 847 normal FTE $(\mathrm{N}=13)$, STIC $(\mathrm{N}=13)$ and HGSC $(\mathrm{N}=12)$. C/EBP $\delta$ expression differs significantly between 848 normal FTE and HGSC tumor samples. D. Representative IHC staining of p53, Ki67 and C/EBP in 849 histologically normal fallopian tube epithelia, STIC and HGSC, respectively. E. Quantification of both $850 \mathrm{C} / \mathrm{EBP} \delta$ and Ki67 levels in FTE $(\mathrm{N}=13)$, STIC $(\mathrm{N}=12)$, and HGSC cases $(\mathrm{N}=12)$ demonstrated an 851 inverse relationship between $\mathrm{C} / \mathrm{EBP} \delta$ and $\mathrm{Ki} 67$ in normal $(\mathrm{p}=0.01)$ and HGSC cases $(\mathrm{p}=0.0001)$. Data 852 are represented as mean $+/-$ SEM and $\mathrm{p}$-values are calculated using student $\mathrm{t}$-tests, two-tailed $(* \mathrm{p}<0.05$; $853 * * * * \mathrm{p}<0.0001)$

Figure 2. Overexpression of C/EBP $\delta$ inhibits proliferation. A. In vitro model of $\mathrm{C} / \mathrm{EBP} \delta$ 856 overexpression in premalignant fallopian tube epithelia. Fallopian tube epithelial cells have a limited life 857 span in-vitro. To generate premalignant cells, FTE were infected with lentivirus containing P53 858 dominant negative mutation $\left(\mathrm{P} 53 \mathrm{DN}^{\mathrm{R} 175 \mathrm{H}}\right)$. Cells with $\mathrm{P} 53 \mathrm{DN}^{\mathrm{R} 175 \mathrm{H}}$ were selected and subsequently 859 immortalized using htert. FTE-P53DN-htert cells were then infected with either control vector or $860 \mathrm{C} / \mathrm{EBP} \delta$. B. Phase contrast microscopy images of a FTE cell line, showing control cells (19-FTE861 P53DN-hTert-ctrl) and C/EBP overxpressing cells (19-FTE-P53DN-hTert-CEBPD-OE) with 862 corresponding images of GFP expression (green) as a marker of successfully infected cells, and C/EBP $\delta$ 863 expression (red) in the nucleus (blue). C. Confirmation of C/EBP $\delta$ protein expression by western blot in 
864 two FTE cell lines, 19 and 57. Pax 8 is a fallopian tube epithelial marker. D. Cell growth measured as 865 population doublings across days in two cell lines, 19 and 57. C/EBP $\delta$ overexpression significantly 866 reduced growth in premalignant FTE. E. To determine cell cycle distribution, BrdU incorporation and 867 DNA content were analyzed by flow cytometry. Representative plot showing percentage distribution of 868 cells across cell cycle control verses C/EBP $\delta$ overexpressing cells using two cell lines, 19 and 57. 869 Statistical significance was set at $\mathrm{p}<0.05$. $\mathrm{C} / \mathrm{EBP} \delta$ overexpression inhibited proliferation by 870 accumulating cells in G1 phase of the cell cycle F. Anchorage independent growth assays (soft agar 871 assays) performed on FTE-Ctrl and FTE-CEBPD-OE cells.

Figure 3. Loss of CEBPD is not necessary for proliferation in MCF7. A. mRNA expression levels measured as fragment per kilobase per million reads (FPKM) of C/EBP $\delta$ and MIB1 (Ki67) across a panel of 42 ovarian cancer cell lines shows an inverse correlation between $\mathrm{C} / \mathrm{EBP} \delta$ and MIB1 expression levels. B. Similarly, C/EBP $\delta$ and MIB1 mRNA expression levels were analyzed across a panel of 54 breast cancer cell lines showing higher levels of C/EBP $\delta$ were associated with lower levels of MIB1 expression. mRNA expression levels are displayed as FPKM. C. Western blot analysis of $\mathrm{C} / \mathrm{EBP} \delta$ expression across six HGSC tumour samples. B-actin is used as a loading control. D. Western 881 Western blot analysis of C/EBP $\delta$ expression in MCF7 cell line transfected with an empty GFP vector 882 (ctrl), $\mathrm{C} / \mathrm{EBP} \delta$ overexpressing vector (CEBPD-OE) and a short hairpin lentiviral construct targeting 883 C/EBP $\delta$ (shCEBPD), respectively. F. Cell growth was measured in MCF7-ctrl cells and MCF7884 shCEBPD. G. Anchorage independent growth assay of MCF7 cells overexpressing C/EBP $\delta$. P-values 885 derived by using an unpaired t-test $(* \mathrm{p}<0.01)$. 
Figure 4. CEBPD overexpression promotes an epithelial cell phenotype with migratory potential.

888 A. Representative IHC staining of $\mathrm{C} / \mathrm{EBP} \delta$, E-cadherin and Vimentin in fallopian tube epithelia from the 889 luteal and follicular phases of the menstrual cycle. B. Quantification of C/EBP $\delta$, E-cadherin and 890 Vimentin protein expression in 21 fallopian tube epithelia cases. C. Macroscopic images were taken 891 using bright field light microscopy of two FTE cell models, 57 and 19 showing control verses C/EBP $\delta$ 892 overexpressing cells. Black arrows indicate elongations from filopodia in C/EBP $\delta$ overexpressing cells. 893 Images were taken at 20x magnification. D. In-vitro analysis of FTE-57 and FTE-19 using western blots 894 showing $\mathrm{C} / \mathrm{EBP} \delta$, e-cadherin, vimentin n-cadherin expression relative to control cells. B-actin is used as 895 a loading control. E. A wound healing assay was performed on FTE-57 and FTE-19 cell lines to 896 demonstrate migration potential. Wound width (uM) was measured against time of experiment 897 (measured in hours).

Figure 5. C/EBPS overexpression downregulates EMT markers and upregulates miRNA involved

901 levels of C/EBPS, and EMT/MET markers, including Snail, Slug, Zeb1, Zeb2 in two FTE cell lines 902 overxpresing $\mathrm{C} / \mathrm{EBP} \delta$ verses control cells. B. A western blot analysis of EMT/MET protein markers 903 perfomed on FTE-57 and FTE-19 overexpressing C/EBP $\delta$ compared to control cells. C. A qPCR of 904 miRNA was performed on both FTE-57 and FTE-19 cells showing the fold change in various miRNAs 905 in cells overexpressing $\mathrm{C} / \mathrm{EBP} \delta$ compared to control cells. Upregulated miRNA (Red) and 906 downregulated (green) were identified $(\mathrm{p}<0.05)$. Data are represented as mean $+/-$ SEM and $\mathrm{p}$-values are 907 calculated using Student t-tests, two-tailed and a 2-way ANOVA. 


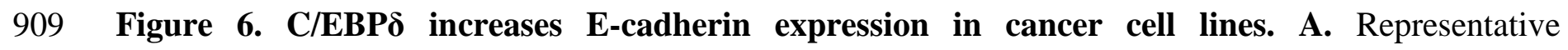

910 macroscopic images of SKOV3 cell line overexpressing C/EBP $\delta$ compared to control, taken using bright

911 field light microscopy at 20× magnification. B. Western blot analysis of $\mathrm{C} / \mathrm{EBP} \delta$, mesenchymal markers

912 vimentin and N-cadherin, epithelial markers E-cadherin and Pax 8, EMT/MET markers, including Zeb1,

913 Zeb2, as well as ER $\alpha$ in SKOV3 cells overexpressing C/EBP $\delta$ compared to controls. C. CEBPD protein

914 expression was compared in three breast cancer cell lines, MCF7, T47D, MDA 231, using western blot

915 analysis. D. Western blot analysis of $\mathrm{C} / \mathrm{EBP} \delta$, mesenchymal marker N-cadherin, epithelial marker E-

916 cadherin, EMT/MET markers, including Zeb1, Snail, as well as ER $\alpha$ Whole-cell lysates in MCF7

917 control cells, C/EBP $\delta$ overexpressing cells and $\mathrm{C} / \mathrm{EBP} \delta$ deficient cells (shCEBPD). B-actin is used as a

918 loading control. E. Images of MCF7 cells overexpressing C/EBP $\delta, \mathrm{C} / \mathrm{EBP} \delta$ deficient cells (shCEBPD)

919 and corresponding controls were taken using bright field light microscope at $20 \times$ magnification. F.

920 Migration assay of MCF7 cells overexpressing $\mathrm{C} / \mathrm{EBP} \delta, \mathrm{C} / \mathrm{EBP} \delta$ deficient cells (shCEBPD) and

921 corresponding controls. Data are represented as mean +/- SEM and p-values are calculated using student

922 t-tests, two-tailed. G. A model showing the role of C/EBP $\delta$ in HGSC development. H. A proposed

923 pathway demonstrating the role of CEBPD in the EMT/MET in the context of fallopian tube epithelia. 
A HGSC
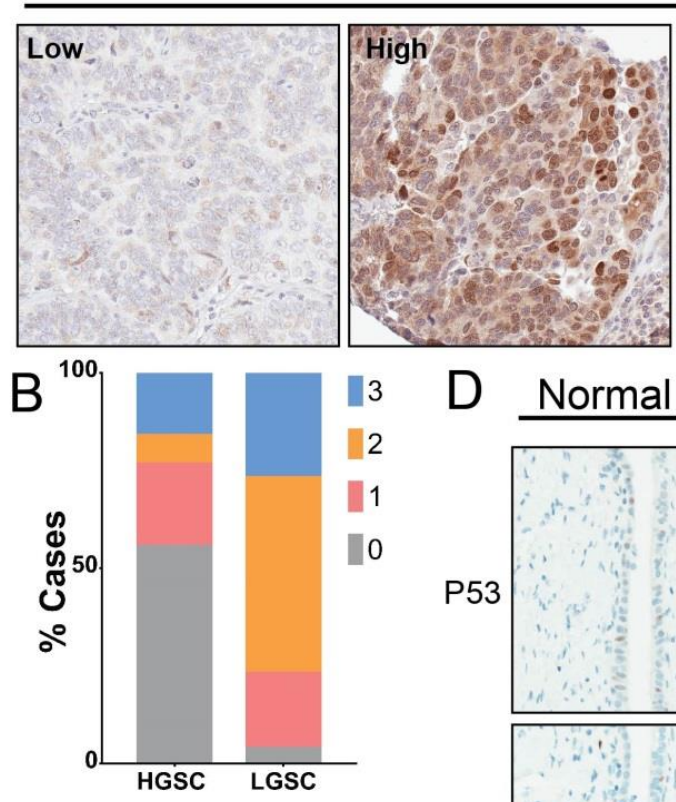

C

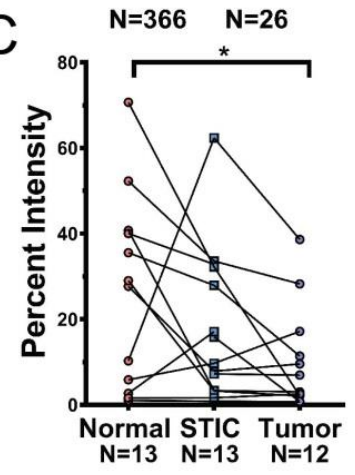

$E$

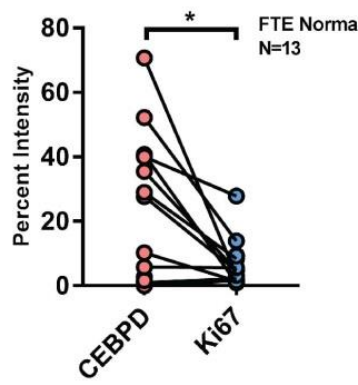

॥2

1

0

\section{CEBPD}

D Normal FTE
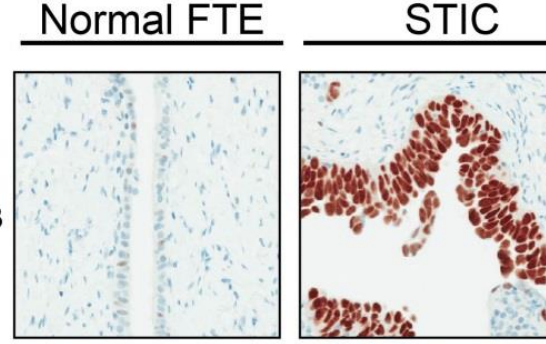

LGSC
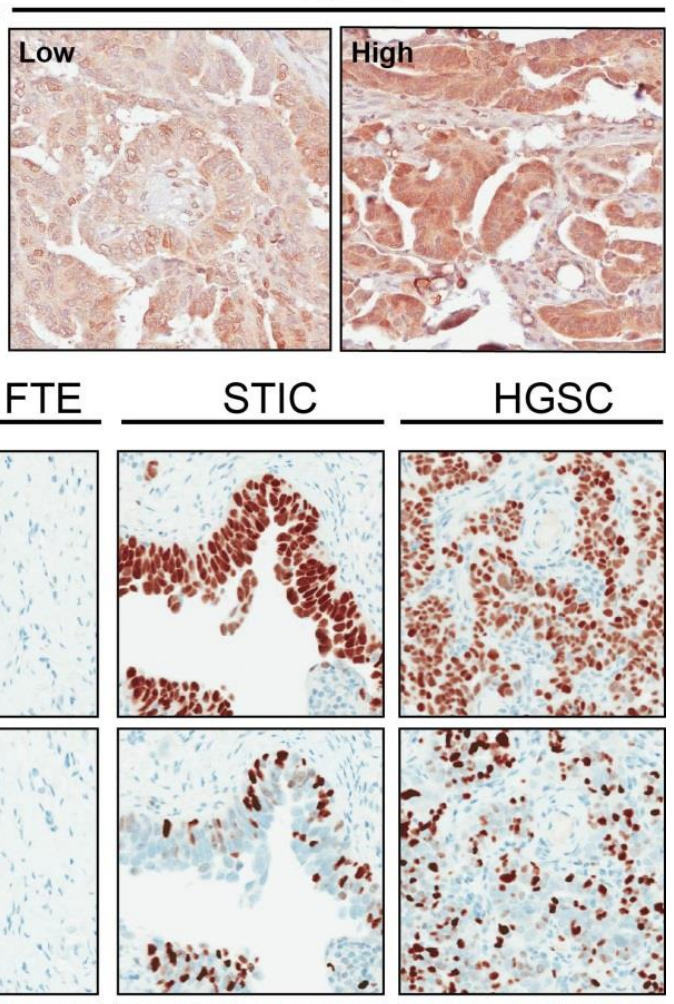

942

943

944 945

Figure 1. C/EBP $\delta$ is differentially expressed between serous histotypes.
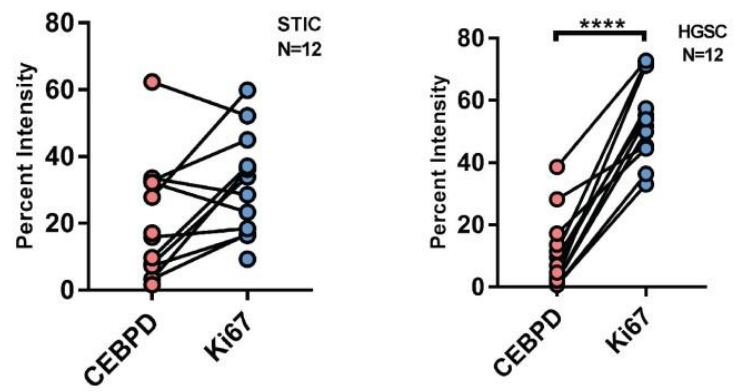
A

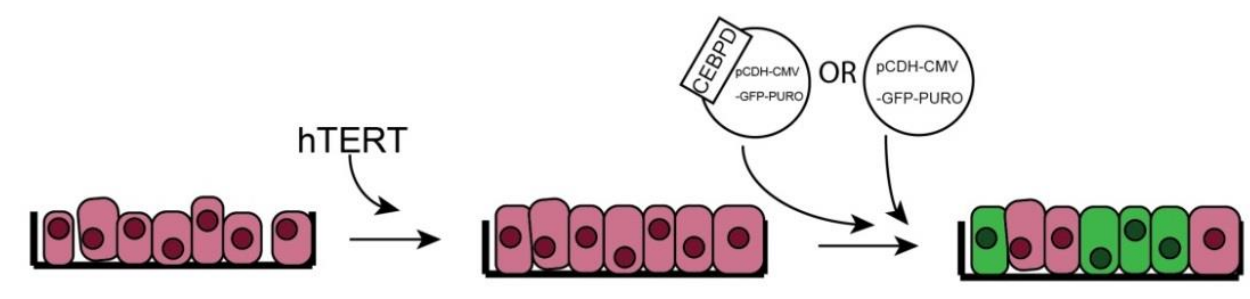

B

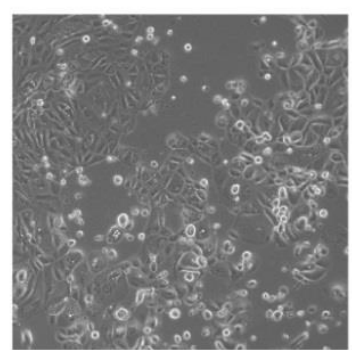

p53DN ${ }^{R 175 H}$

D
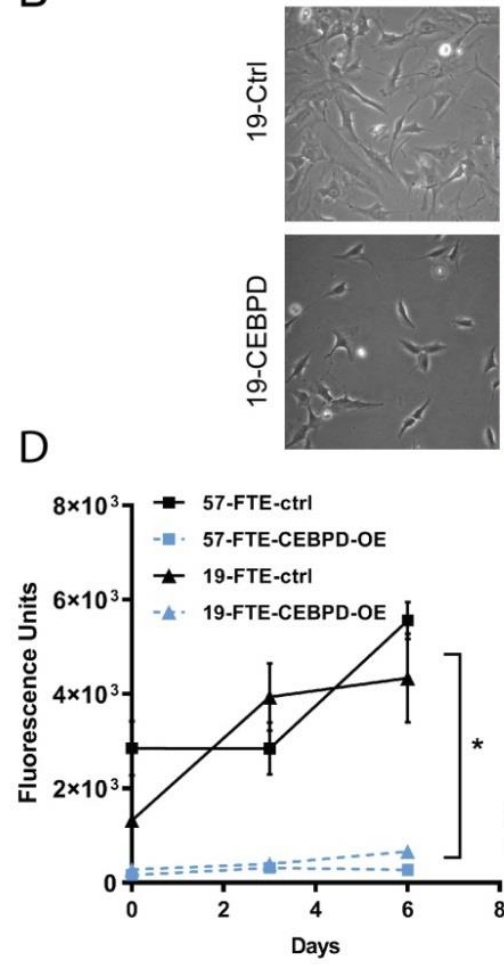

F

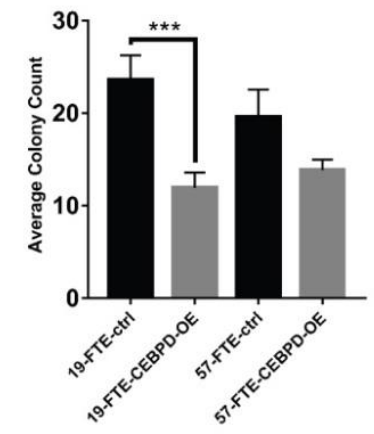

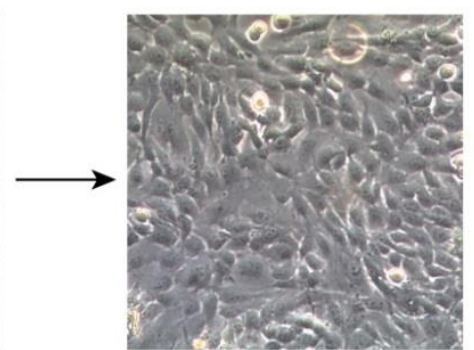

p53DN ${ }^{R 175 H}+$ hTERT
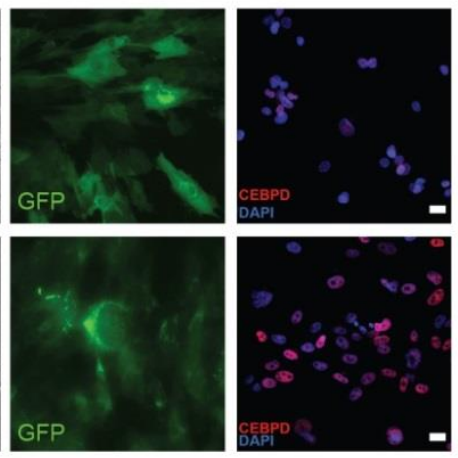

$\mathrm{E}$
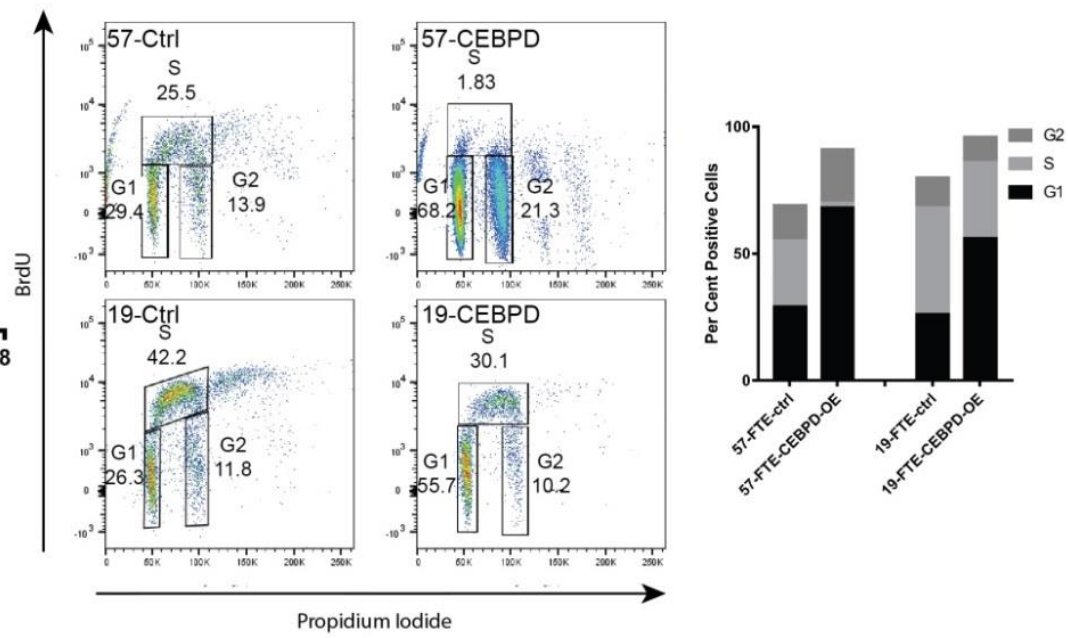

Figure 2. Overexpression of C/EBP $\delta$ inhibits proliferation. 
A B
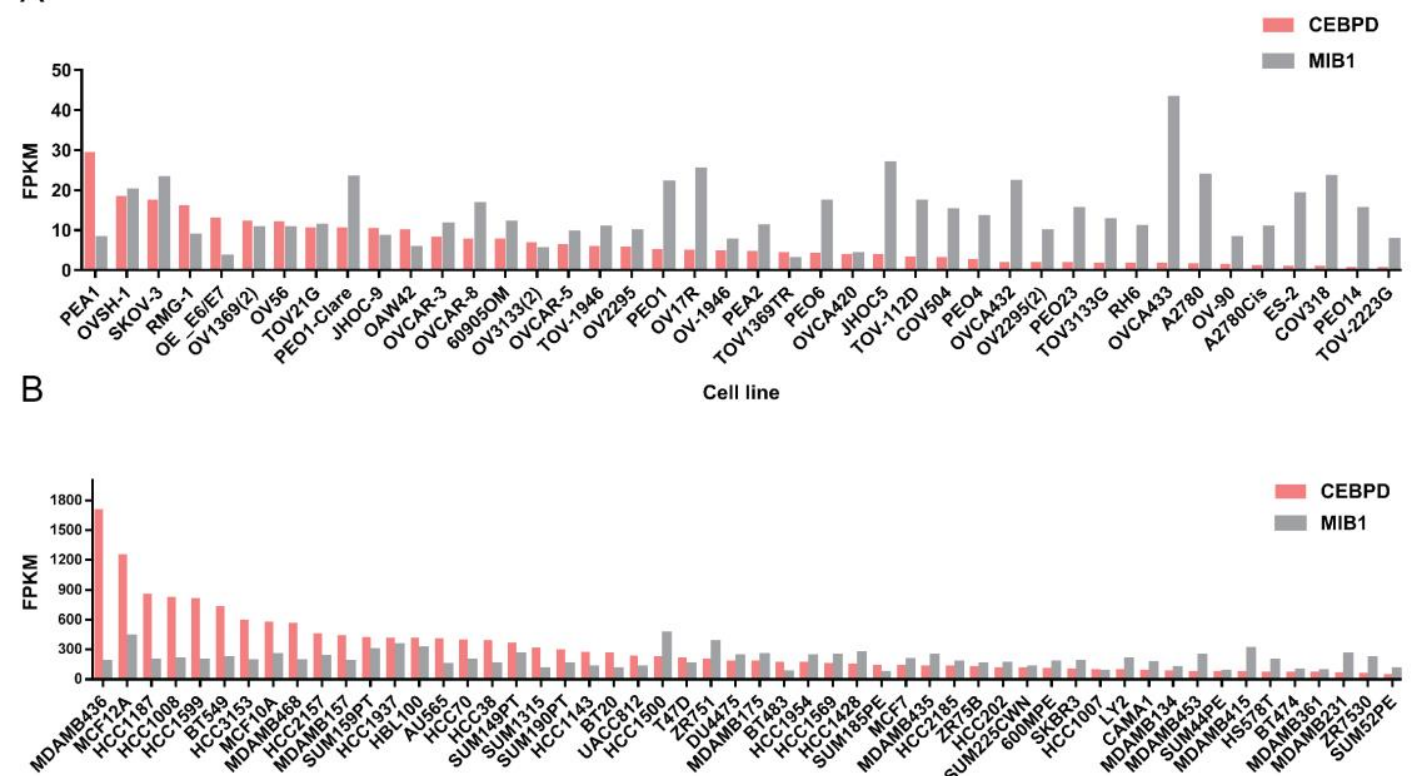
Cell line
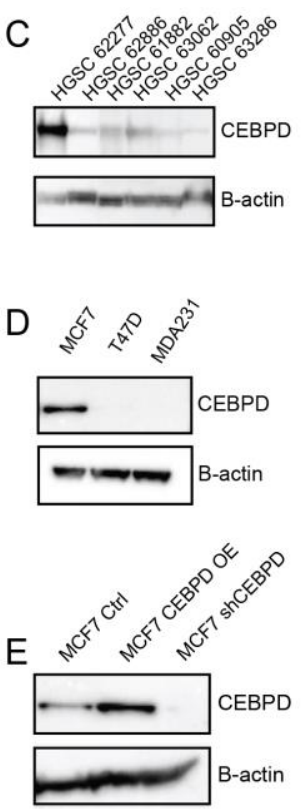

F

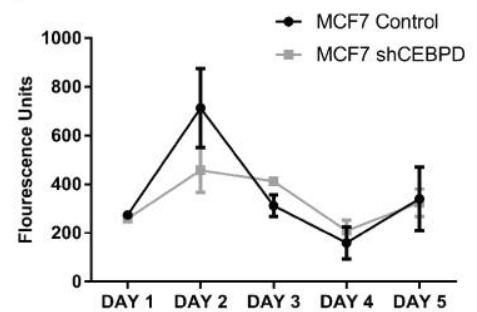

G

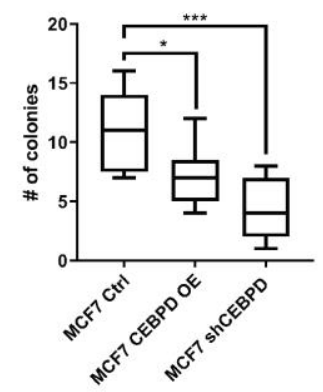

949

950

951

952

Figure 3. Loss of CEBPD is not necessary for proliferation in MCF7. 
A
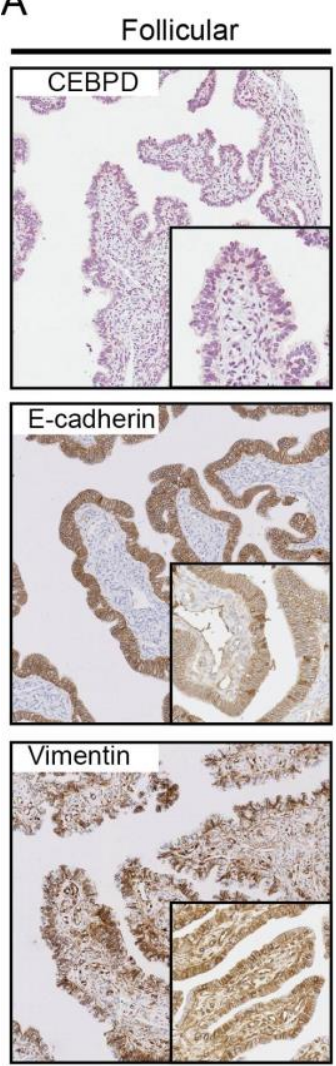

Luteal
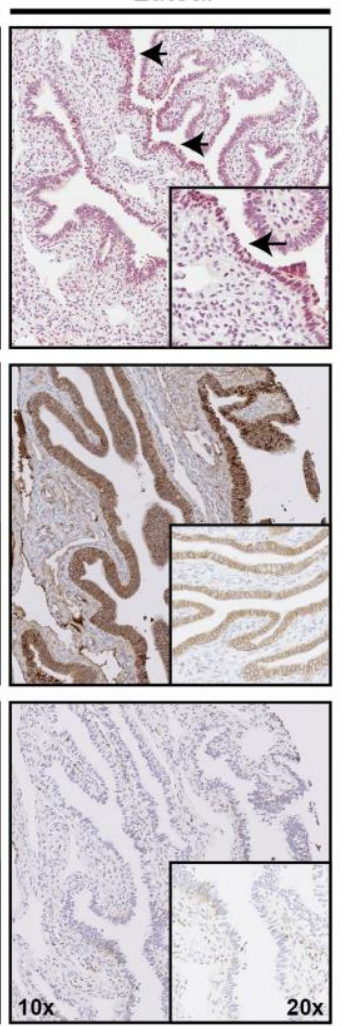

B

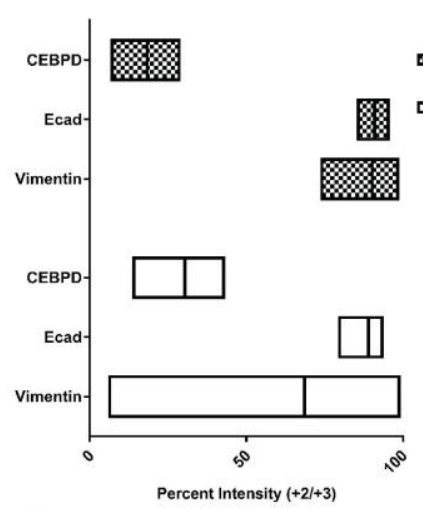

D

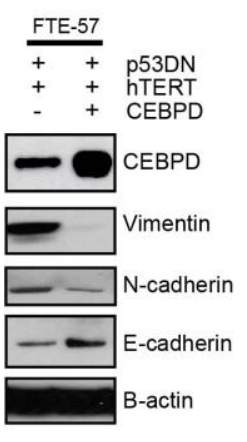

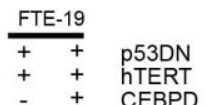
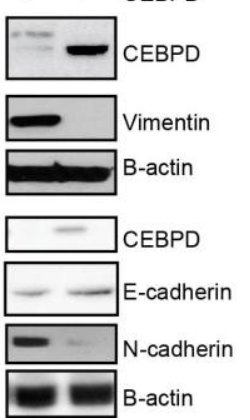

C

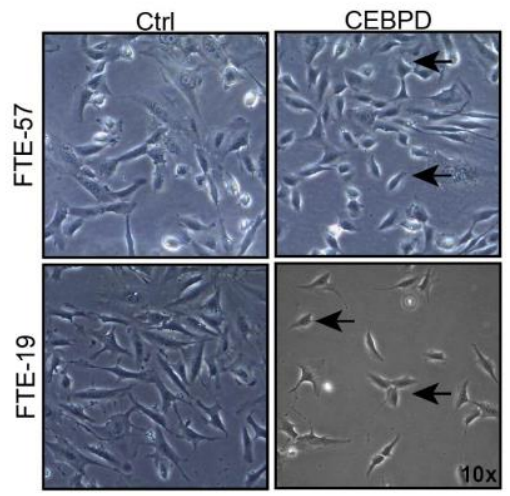

$\mathrm{E}$

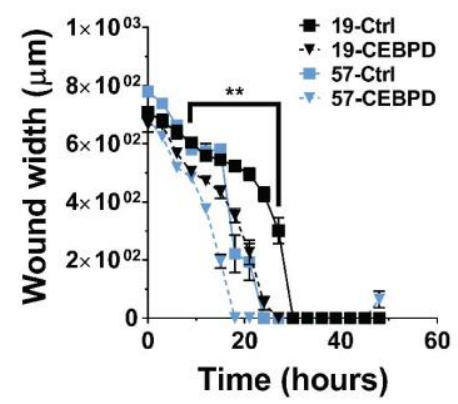


A
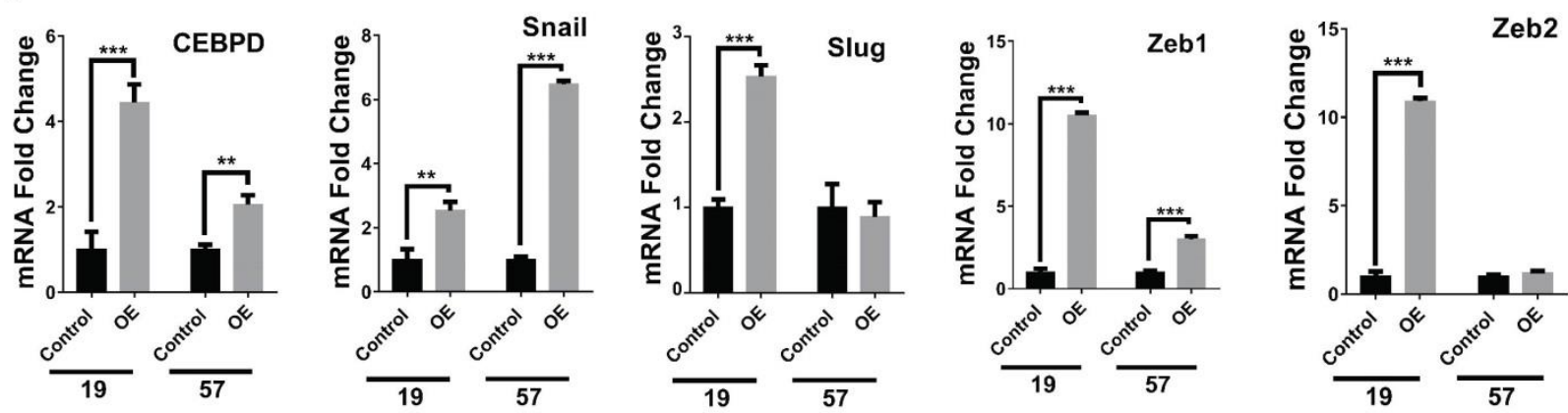

B
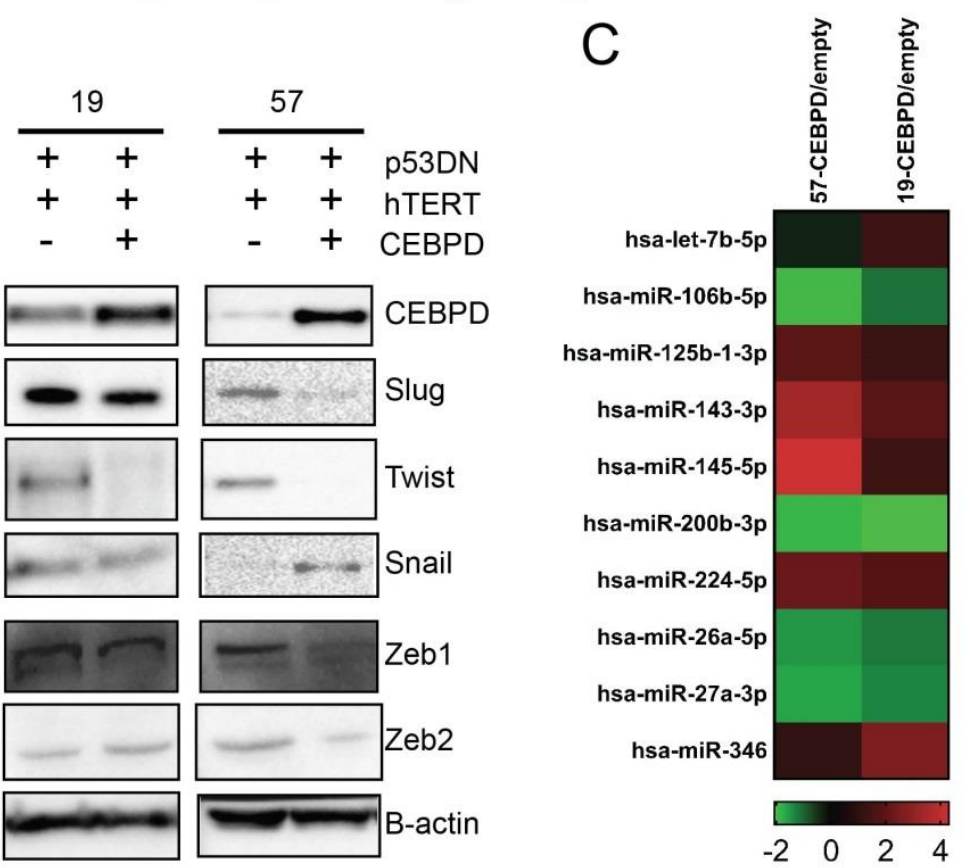

$+++\quad+$ hTERT

- $+\quad+\quad+$ CEBPD
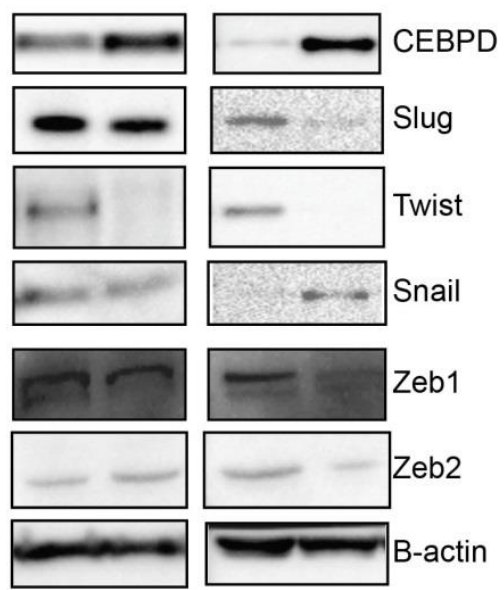

Figure 5. C/EBPס overexpression downregulates EMT markers and upregulates miRNA involved 


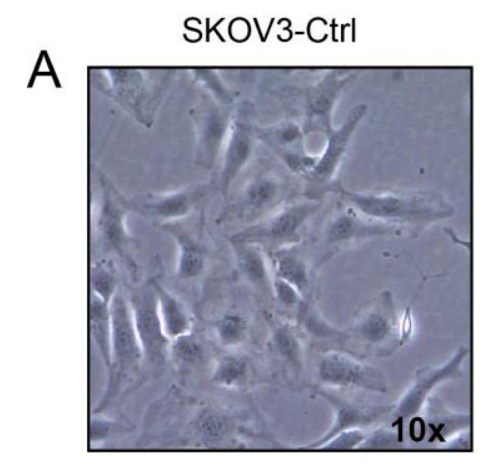

B

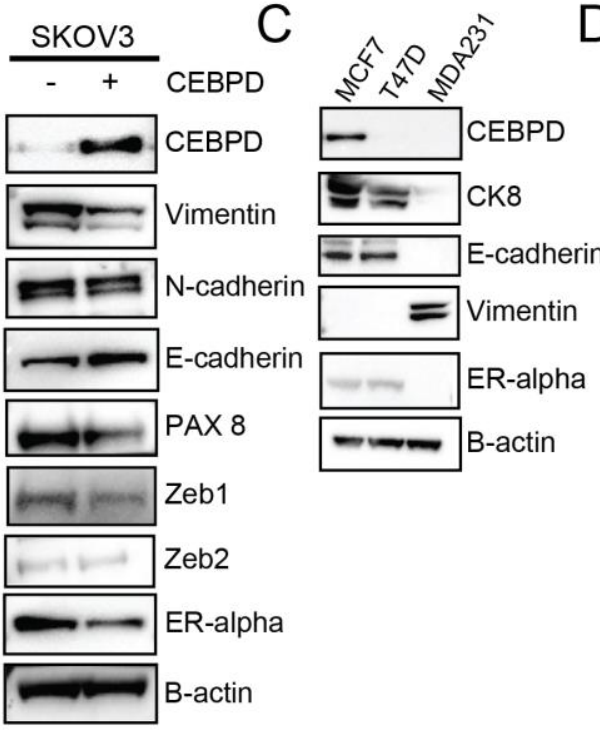

SKOV3-CEBPD

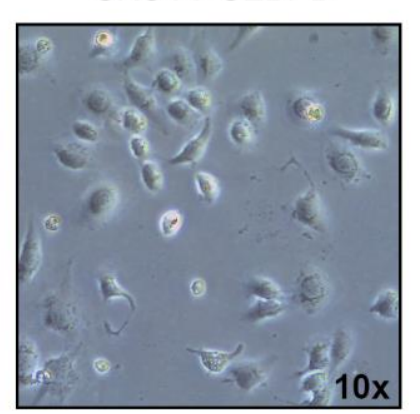

D

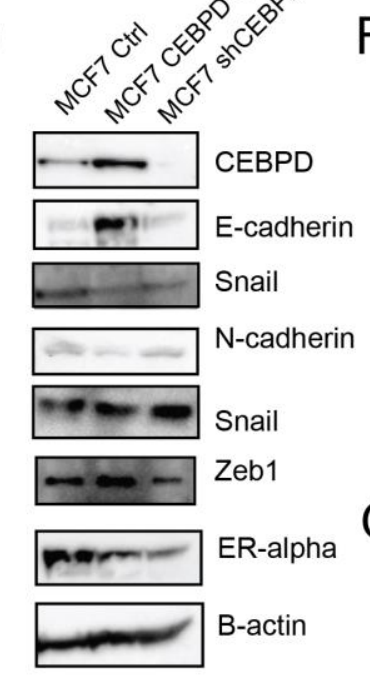

(2)
E

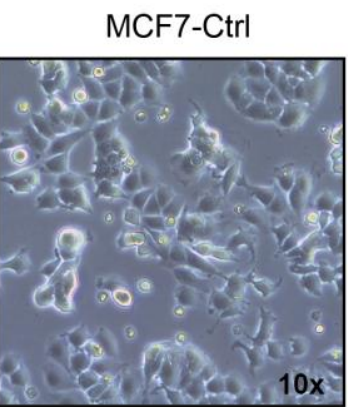

F Transwell Migration Assay (200-
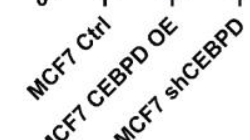

$\mathrm{G}$
MCF7-CEBPD

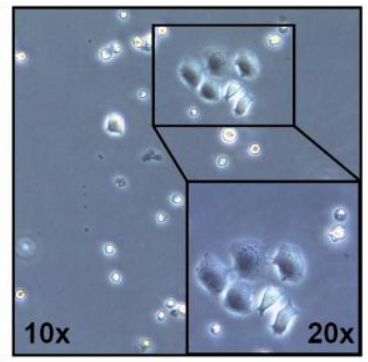

$\mathrm{H} \longrightarrow$
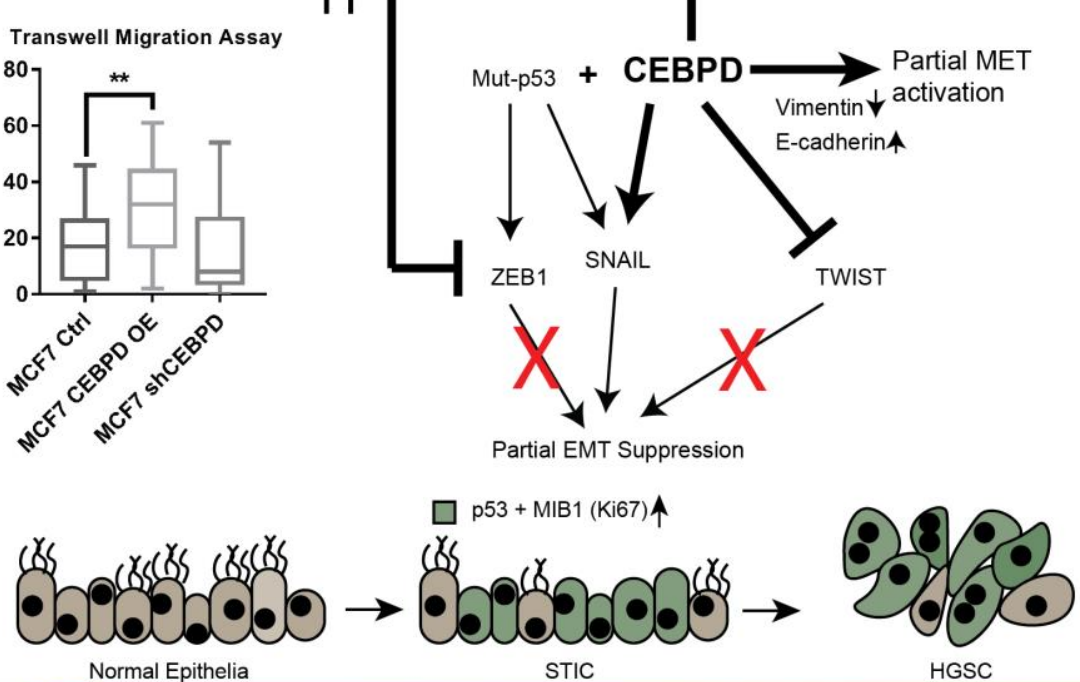

Figure 6. C/EBP $\delta$ increases E-cadherin expression in cancer cell lines. 